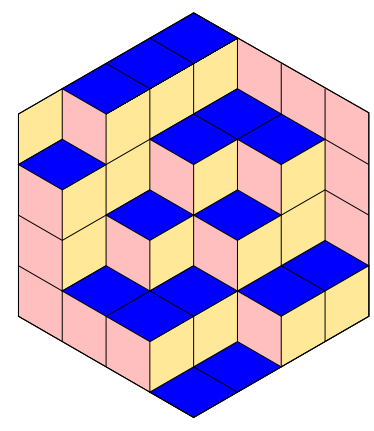

Per Alexandersson \& Joakim Uhlin

Cyclic sieving, skew Macdonald polynomials and Schur positivity

Volume 3, issue 4 (2020), p. 913-939.

<http://alco.centre-mersenne.org/item/ALCO_2020__3_4_913_0>

(C) The journal and the authors, 2020.

Some rights reserved.

(c) BY This article is licensed under the

Creative Commons AtTribution 4.0 International License.

http://creativecommons.org/licenses/by/4.0/

Access to articles published by the journal Algebraic Combinatorics on the website http://alco.centre-mersenne.org/ implies agreement with the Terms of Use (http://alco.centre-mersenne.org/legal/).

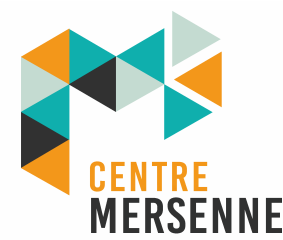

Algebraic Combinatorics is member of the Centre Mersenne for Open Scientific Publishing www.centre-mersenne.org 


\title{
Cyclic sieving, skew Macdonald polynomials and Schur positivity
}

\author{
Per Alexandersson \& Joakim Uhlin
}

\begin{abstract}
When $\lambda$ is a partition, the specialized non-symmetric Macdonald polynomial $\mathrm{E}_{\lambda}(\mathbf{x} ; q ; 0)$ is symmetric and related to a modified Hall-Littlewood polynomial. We show that whenever all parts of the integer partition $\lambda$ are multiples of $n$, the underlying set of fillings exhibit the cyclic sieving phenomenon (CSP) under an $n$-fold cyclic shift of the columns. The corresponding CSP polynomial is given by $\mathrm{E}_{\lambda}(\mathbf{x} ; q ; 0)$. In addition, we prove a refined cyclic sieving phenomenon where the content of the fillings is fixed. This refinement is closely related to an earlier result by B. Rhoades.

We also introduce a skew version of $\mathrm{E}_{\lambda}(\mathbf{x} ; q ; 0)$. We show that these are symmetric and Schur positive via a variant of the Robinson-Schenstedt-Knuth correspondence and we also describe crystal raising and lowering operators for the underlying fillings. Moreover, we show that the skew specialized non-symmetric Macdonald polynomials are in some cases vertical-strip LLT polynomials. As a consequence, we get a combinatorial Schur expansion of a new family of LLT polynomials.
\end{abstract}

\section{INTRODUCTION}

The cyclic sieving phenomenon (CSP), introduced by V. Reiner, D. Stanton and D. White [35], is currently an active research topic, see e.g. [11, 33, 38, 41]. In this article, we provide families of cyclic sieving on tableaux related to certain specializations of non-symmetric Macdonald polynomials. This settles an earlier conjecture by the authors presented in [48]. The non-symmetric Macdonald polynomials are in our case closely related to the transformed Hall-Littlewood functions and Kostka-Foulkes polynomials, previously studied in the CSP context by B. Rhoades [37]. The family of polynomials we study is the specialization of the non-symmetric Macdonald polynomials $\mathrm{E}_{\lambda}\left(x_{1}, \ldots, x_{m} ; q, t\right)$ when $\lambda$ is an integer partition and $t=0$. They can be defined as a weighted sum over certain fillings of the Young diagram $\lambda$. We denote this set of fillings $\operatorname{COF}(\lambda, m)$, which is defined Section 2.8.

1.1. MaIn RESULTS. For an integer partition $\lambda=\left(\lambda_{1}, \ldots, \lambda_{\ell}\right)$, we let $n \lambda$ denote the partition $\left(n \lambda_{1}, \ldots, n \lambda_{\ell}\right)$. We show that there is a natural action $\phi$ on the fillings $\operatorname{COF}(n \lambda, m)$ where each block of $n$ consecutive columns is cyclically rotated one step. Consequently $\phi$ generates a $C_{n}$-action on $\operatorname{COF}(n \lambda, m)$. In Theorem 3.2, we prove that for every $n, m \in \mathbb{N}_{+}$and integer partition $\lambda$, the triple

$$
\left(\mathrm{COF}(n \lambda, m),\langle\phi\rangle, \mathrm{E}_{n \lambda}\left(1^{m} ; q, 0\right)\right)
$$

Manuscript received 15th August 2019, revised 25th March 2020, accepted 12th April 2020.

KeYwords. Cyclic sieving, Macdonald polynomials, LLT polynomials, crystals, Schur-positivity. 
exhibits the cyclic sieving phenomenon. Moreover, as $\lambda$ is held fixed and $n=$ $1,2,3, \ldots$, this family is a Lyndon-like family, a notion by P. Alexandersson, S. Linusson and S. Potka [3] (see also [19]) meaning that fixed points in $\operatorname{COF}(n \lambda, m)$ under $\phi^{k}$ are in natural bijection with the elements in $\operatorname{COF}\left(\frac{n}{k} \lambda, m\right)$ whenever $k \mid n$. When $\lambda=(1)$, this phenomenon reduces to a classical cyclic sieving phenomenon on words of length $n$ in the alphabet $[m]$, see Example 2.10 below. A skew version of (1) is given in Theorem 5.10.

We also prove a refined cyclic sieving phenomenon. Let $\operatorname{COF}(n \lambda, \nu)$ denote the set of coinversion-free fillings with shape $n \lambda$ and content $\nu$. In Theorem 4.3, we show that

$$
\left(\operatorname{COF}(n \lambda, \nu),\langle\phi\rangle,\left[\mathrm{m}_{\nu}\right] \mathrm{E}_{n \lambda}(\mathbf{x} ; q, 0)\right)
$$

exhibits the cyclic sieving phenomenon. When $\lambda=(n)$, we recover the cyclic sieving phenomenon on words of length $n$ with content $\nu$, and

$$
\left[\mathrm{m}_{\nu}\right] \mathrm{E}_{(n)}(\mathbf{x} ; q, 0)=\left[\begin{array}{l}
n \\
\nu
\end{array}\right]_{q}
$$

a $q$-multinomial coefficient. We remark that if we take $\lambda=\left(1^{k}\right),\left[\mathrm{m}_{\nu}\right] \mathrm{E}_{n \lambda}(\mathbf{x} ; q, 0)$ in (2) can be seen as a $q$-analogue of $n$-tuples of $k$-subsets of $[m]$ with content $\nu$.

In Section 5 we introduce a skew version of $\mathrm{E}_{\lambda}(\mathbf{x} ; q, 0)$ and prove that these are symmetric and Schur positive. We provide an explicit Schur expansion using a generalization of charge in Theorem 5.7. As an application, in Theorem 7.3 we obtain a combinatorial Schur expansion of a certain family of vertical-strip LLT polynomials, which has not been considered before. Combining Theorem 7.3 and Theorem 5.7, we have the following main result.

THEOREM 1.1. Let $\lambda / \mu$ be a skew shape such that no column contains more than two boxes. Let $\boldsymbol{\nu}$ be the tuple of skew shapes such that $\nu_{j}$ is the vertical strip $1^{\lambda_{j}} / 1^{\mu_{j}}$ and set $\alpha_{i}:=\lambda_{i}-\mu_{i}$. Then

$$
\operatorname{LLT}_{\boldsymbol{\nu}}(\mathbf{x} ; q)=q^{\operatorname{mininv}(\boldsymbol{\nu})} \sum_{\rho \vdash\left|\lambda^{\prime} / \mu^{\prime}\right|} \mathrm{s}_{\rho^{\prime}}(\mathbf{x}) \sum_{T \in \operatorname{SSYT}(\rho, \alpha)} q^{\operatorname{charge}_{\mu}(T)}
$$

where charge $_{\mu}$ is a natural generalization of the charge statistic defined in Definition 5.2, and $\operatorname{mininv}(\boldsymbol{\nu})$ is a simple statistic that only depends on the tuple $\boldsymbol{\nu}$ given in Equation (28).

The paper is structured as follows. In Section 2, we define the cyclic sieving phenomenon and give a brief overview of the relevant symmetric functions. In Section 3 , we give a proof of the CSP in (1), and in Section 4 we prove (2). In Section 5 we introduce the skew specialized Macdonald polynomials and give the Schur expansion of these. In Section 6, we define crystal operators on the related skew coinversion-free fillings and thus gives an alternative proof of the crystal structure given in [9]. Finally, we prove a result in Section 7 which implies Theorem 1.1.

We note that some of the results in this paper are based on earlier work done in the second author's master's thesis [48].

\section{Preliminaries}

\subsection{Partitions And COMPOSitions.}

Definition 2.1. Let $n$ and $\ell$ be natural numbers. $A$ weak composition $\lambda$ of $n$ into $\ell$ parts is defined to be an $\ell$-tuple $\lambda=\left(\lambda_{1}, \ldots, \lambda_{\ell}\right)$ of non-negative integers such that $\lambda_{1}+\cdots+\lambda_{\ell}=n$. We say that the numbers $\lambda_{1}, \ldots, \lambda_{\ell}$ are the parts of $\lambda$. If all parts of $\lambda$ are positive, we say that $\lambda$ is a composition, and we write $\lambda \vDash n$. If $\lambda$ has multiple parts of the same size, we may suppress them using exponents. As an 
example, $(7,7,0,1,1,1,4,4,4,4)$ may be expressed as $\left(7^{2}, 0,1^{3}, 4^{4}\right)$. We write $m_{j}(\lambda)$ for the number of parts of $\lambda$ equal to $j$ and $n \lambda:=\left(n \lambda_{1}, \ldots, n \lambda_{\ell}\right)$ for $n \in \mathbb{N}$. If further $\lambda_{1} \geqslant \lambda_{2} \geqslant \cdots \geqslant \lambda_{\ell}$, then $\lambda$ is a partition of $n$, and denote this by $\lambda \vdash$ $n$. The parts of $\lambda$ are the positive entries of $\lambda$. The length of $\lambda$ is the number of parts and is denoted $\ell(\lambda)$. We identify partitions that only differ by trailing zeros, so $(4,2,2,1,0,0,0)=(4,2,2,1,0)=(4,2,2,1)$ as partitions. There is one unique partition of 0 , namely $\varnothing$ which is referred to as the empty partition.

Note that in some cases, the word parts is ambiguous. When $\lambda$ is a weak composition, a part can be zero whereas when $\lambda$ is a partition, a part must be a positive integer. This conflicting terminology is unfortunately very standard, see e.g. [45].

\subsection{Semistandard Young tableau.}

Definition 2.2. Let $\lambda=\left(\lambda_{1}, \ldots, \lambda_{\ell}\right) \vdash n$. The Young diagram of $\lambda$ is defined as the set $\left\{(i, j) \in \mathbb{Z}^{2}: 1 \leqslant i \leqslant \lambda_{j}\right\}$. Geometrically ${ }^{(1)}$ we think of this diagram as a set of $n$ boxes with $\ell$ left-justified rows and $\lambda_{i}$ boxes in row $i$. The box in position $(i, j)$ is the box in the $i^{\text {th }}$ row and $j^{\text {th }}$ column. We use the notation $\lambda$ to both refer to the partition and to the Young diagram described by $\lambda$. The number of boxes in a diagram $\lambda$ is denoted $|\lambda|$.

Define the conjugate of $\lambda$, denoted $\lambda^{\prime}$, to be the Young diagram obtained by transposing $\lambda$ where the boxes may be seen as matrix entries. We write $\lambda^{\prime}=\left(\lambda_{1}^{\prime}, \ldots, \lambda_{\ell}^{\prime}\right)$. If $\lambda$ is a partition on the form $\lambda=a^{b}$, then $\lambda$ is a rectangular Young diagram. Throughout this article, all the diagrams are displayed in English notation, using matrix coordinates, with a few exceptions in Section $\%$.
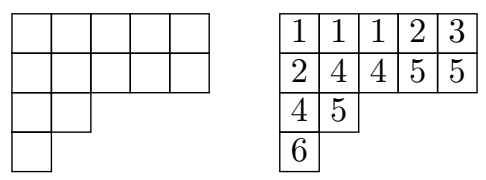

Figure 1. To the left: A Young diagram of shape $\lambda=(5,5,2,1)$. To the right: A semistandard Young tableau of shape $\lambda$.

Definition 2.3. Let $\lambda$ be a Young diagram. A filling of $\lambda$ is a map $T: \lambda \rightarrow \mathbb{N}_{+}$and a semistandard Young tableau (SSYT) is a filling of $\lambda$ such that in each row the entries are weakly increasing and in each column the entries are strictly increasing. The set of all semistandard Young tableaux of shape $\lambda$ is denoted $\operatorname{SSYT}(\lambda)$ and we let $\operatorname{SSYT}(\lambda, \mu)$ the the set of such SSYT where the number of entries equal to $i$ is given by $\mu_{i}$.

Let $T$ be a semistandard Young tableau. Define the reading word of $T$, denoted $\mathrm{rw}(T)$, as the word obtained by reading the entries $T$ from the bottom row to the top row and in each row from left to right. For example, the semistandard Young tableau in Figure 1 has reading word $\mathrm{rw}(T)=6452445511123$. We let $\mathbf{x}^{T}:=\prod_{j} x_{j}^{m_{j}(T)}$ where $m_{j}(T)$ is the number of entries in $T$ equal to $j$. The semistandard Young tableau $T$ in Figure 1 gives $\mathbf{x}^{T}=x_{1}^{3} x_{2}^{2} x_{3} x_{4}^{3} x_{5}^{3} x_{6}$.

\footnotetext{
${ }^{(1)}$ Note, we use the computer-friendly matrix indexing (row, column), which has the advantage that it is also invariant under English/French convention.
} 
There are several equivalent ways to define the Schur functions but the following is the most useful for our purposes. We let the Schur function indexed by the integer partition $\lambda$ be defined as

$$
\mathrm{s}_{\lambda}(\mathbf{x}):=\sum_{T \in \operatorname{SSYT}(\lambda)} \mathbf{x}^{T}
$$

2.3. $q$-ANALOGUES. A $q$-analogue of a certain expression is a rational function in the variable $q$ from which we can obtain the original expression by letting $q \rightarrow 1$.

Definition 2.4. Let $n \in \mathbb{N}$. Define the $q$-analogue of $n$ as $[n]_{q}:=1+q+\cdots+q^{n-1}$. Furthermore, define the $q$-factorial of $n$ as $[n]_{q} !:=[n]_{q}[n-1]_{q} \cdots[1]_{q}$. Lastly, the $q$-binomial coefficient is defined as

$$
\left[\begin{array}{l}
n \\
k
\end{array}\right]_{q}:=\frac{[n]_{q} !}{[n-k]_{q} ![k]_{q} !} \text { if } n \geqslant k \geqslant 0 \text {, and } 0 \text { otherwise. }
$$

Theorem 2.5 ( $q$-Lucas theorem, see e.g. [39]). Let $n, k \in \mathbb{N}$. Let $n_{1}, n_{0}, k_{1}, k_{0}$ be the unique natural numbers satisfying $0 \leqslant n_{0}, k_{0} \leqslant d-1$ and $n=n_{1} d+n_{0}, k=k_{1} d+k_{0}$. Then

$$
\left[\begin{array}{l}
n \\
k
\end{array}\right]_{q} \equiv\left(\begin{array}{l}
n_{1} \\
k_{1}
\end{array}\right)\left[\begin{array}{l}
n_{0} \\
k_{0}
\end{array}\right]_{q} \quad\left(\bmod \Phi_{d}(q)\right)
$$

where $\Phi_{d}(q)$ is the $d^{\text {th }}$ cyclotomic polynomial. In particular, we have

$$
\left[\begin{array}{l}
n \\
k
\end{array}\right]_{\xi}=\left(\begin{array}{l}
n_{1} \\
k_{1}
\end{array}\right)\left[\begin{array}{l}
n_{0} \\
k_{0}
\end{array}\right]_{\xi}
$$

if $\xi$ is a primitive $d^{\text {th }}$ root.

Theorem 2.5 will be used in later sections.

2.4. Charge and Kostka-Foulkes polynomials. We shall briefly describe the charge statistic and the related Kostka-Foulkes polynomials $K_{\lambda \mu}(q)$ appearing in later sections. This combinatorial model was first described by A. Lascoux and M. Schützenberger [30]. For a permutation $\sigma \in S_{k}$, let $\operatorname{Des}(\sigma):=\left\{i \in[k-1]: \sigma_{i+1}<\sigma_{i}\right\}$, the major index be defined as maj $(\sigma):=\sum_{j \in \operatorname{Des}(\sigma)} j$, and let $\operatorname{rev}(\sigma):=\left(\sigma_{n}, \sigma_{k-1}, \ldots, \sigma_{1}\right)$ be the reverse. We can now introduce the notion of charge of a permutation.

$$
\operatorname{charge}(\sigma):=\operatorname{maj}\left(\operatorname{rev}\left(\sigma^{-1}\right)\right)=\sum_{i \notin \operatorname{Des}\left(\sigma^{-1}\right)}(k-i) .
$$

For example,

$$
\operatorname{charge}(198423765)=\operatorname{maj}(\operatorname{rev}(156498732))=\operatorname{maj}(237894651)=20 .
$$

We note that our way of defining charge is different from [30].

Given a word $w$ with content $\mu \vdash n$, we partition its entries into standard subwords as follows. Start from the right of $w$ and mark the first occurrence of 1 . Proceed to the left, and mark the first occurrence of 2 , then 3 and so on, wrapping around the end if nessecary, until $\mu_{1}^{\prime}$ entries have been marked. This subword is the first standard subword of $w$. Remove this subword, and repeat the process to find the second standard subword, of length $\mu_{2}^{\prime}$.

For example, the first standard subword in $w=21123543411223$ has been circled.

$$
2,1,1,2 \text {, 3, (5), 4, 3, 4, 1, (1), 2, 2, 3. }
$$

In total, we have four standard subwords in $w$, with corresponding charge values

$$
\operatorname{charge}(25413)=3, \quad \operatorname{charge}(2431)=2, \quad \operatorname{charge}(132)=2, \quad \text { charge }(12)=1,
$$


and we define charge $(w)$ as the sum of the charge values of the standard subwords. In the example above, $\operatorname{charge}(w)=8$.

Recall the definition of the reading word $\operatorname{rw}(T)$ of a semistandard Young tableau from Definition 2.3. We then define charge $(T):=$ charge $(\operatorname{rw}(T))$ and the KostkaFoulkes polynomial $K_{\lambda \mu}(q)$ may be computed as

$$
K_{\lambda \mu}(q)=\sum_{T \in \operatorname{SSYT}(\lambda, \mu)} q^{\operatorname{charge}(T)} .
$$

ExAmple 2.6 (Computing a Kostka-Foulkes polynomial). Consider the case $\lambda=421$, $\mu=3211$. There are four tableaux in $\operatorname{SSYT}(\lambda, \mu)$. Below, these are displayed, each with the list of standard subwords and corresponding charge values.

\begin{tabular}{|c|c|c|c|c|c|c|c|c|c|c|c|}
\hline 1 & 1 & \begin{tabular}{l|l}
1 & 4 \\
\end{tabular} & 1 & \begin{tabular}{|l|l|}
1 & 1 \\
\end{tabular} & 3 & 1 & 1 & \begin{tabular}{l|l}
1 & 2 \\
\end{tabular} & 1 & 1 & \begin{tabular}{l|l}
1 & 2 \\
\end{tabular} \\
\hline 2 & 2 & & 2 & 2 & & 2 & 4 & & 2 & 3 & \\
\hline 3 & & & 4 & & & 3 & & & 4 & & \\
\hline & $\begin{array}{l}14, \\
1+0\end{array}$ & $\begin{array}{l}21,1 \\
+0\end{array}$ & & $\begin{array}{l}13,21 \\
2+0+0\end{array}$ & & & & $\begin{array}{l}12,1 \\
+0\end{array}$ & & $\begin{array}{l}31, \\
2+1\end{array}$ & $\begin{array}{l}12,1 \\
+0\end{array}$ \\
\hline
\end{tabular}

Hence, $K_{\lambda \mu}(q)=q+2 q^{2}+q^{3}$.

\subsection{CyCliC SIEVING.}

Definition 2.7 (Cyclic sieving, see [35]). Let $X$ be a set of combinatorial objects and $C_{n}=\langle g\rangle$ be the cyclic group of order $n$ acting on $X$, with $g$ as a generator. Let $f(q) \in \mathbb{N}[q]$ be a polynomial with non-negative integer coefficients. We say that the triple $\left(X, C_{n}, f(q)\right)$ exhibits the cyclic sieving phenomenon, (CSP) if for all $d \in \mathbb{Z}$,

$$
\#\left\{x \in X: g^{d} \cdot x=x\right\}=f\left(\xi^{d}\right)
$$

where $\xi$ is a primitive $n^{\text {th }}$ root of unity.

Note that it follows immediately from the definition that $\# X=f(1)$. In practice, the group action of $C_{n}$ on $X$ and the polynomial $f(q)$ is almost always natural in some sense. The group action could be some form of rotation or cyclic shift of the elements of $X$. The polynomial usually has a closed form and is also typically the generating polynomial for some combinatorial statistic defined on $X$.

ExAMPLE 2.8 ( $k$-subset CSP, see [35]). Let $\left(\begin{array}{c}{[n]} \\ k\end{array}\right)$ be the set of $k$-subsets of $[n]$. Suppose that $C_{N}$ is generated by a permutation $\sigma \in S_{n}$, where the cycles of $\sigma$ consists of $N$ cycles and one or zero singletons. Let $C_{N}$ act on $[n]$ in the natural way (this is referred to as $C_{N}$ acting nearly freely on $\left.[n]\right)$. Then $\left(\left(\begin{array}{c}{[n]} \\ k\end{array}\right), C_{N},\left[\begin{array}{c}n \\ k\end{array}\right]_{q}\right)$ exhibits the cyclic sieving phenomenon.

In Table 1 we summarize some of the most famous and relevant instances of cyclic

\begin{tabular}{|c|c|c|c|}
\hline Set & Group action & Polynomial & Reference \\
\hline$k$-subsets of $[n]$ & Nearly free action & & {$[35]$} \\
\hline Words with content $\alpha$ & Cyclic shift & & {$[35]$} \\
\hline Non-cross. perf. matchings & Rotation & $\frac{1}{[n+1]_{q}}\left[\begin{array}{c}2 n \\
n\end{array}\right]_{q}$ & {$[35]$} \\
\hline $\operatorname{SYT}\left(n^{m}\right)$ & Promotion & $f^{\lambda}(q)$ & {$[36]$} \\
\hline 01-matrices & Shift rows/columns & See Theorem 4.1 & {$[37]$} \\
\hline
\end{tabular}
sieving. For a more comprehensive list, see B. Sagan's article [40]. One of the main

TABLE 1. A few known instances of cyclic sieving. 
results of this paper, Theorem 3.2, is a generalization of the first instance of cyclic sieving in Table 1 and it is also closely related to the last instance in the table.

The situation is even more interesting when different instances of the cyclic sieving phenomenon are related in a certain fashion.

DEFINITION 2.9 (Lyndon-like CSP, [3]). Let $\left\{X_{n}\right\}_{n=1}^{\infty}$ be a family of combinatorial objects with a cyclic group action $C_{n}$ acting on $X_{n}$. Furthermore, let $\left\{f_{n}(q)\right\}_{n=1}^{\infty}$ be a sequence of polynomials in $\mathbb{N}[q]$, such that for each $n=1,2, \ldots$, the triple $\left(X_{n}, C_{n}, f_{n}(q)\right)$ exhibits the cyclic sieving phenomenon. We say that the family of triples $\left\{\left(X_{n}, C_{n}, f_{n}(q)\right)\right\}_{n=1}^{\infty}$ is Lyndon-like if $f_{n / d}(1)=f_{n}\left(e^{\frac{2 \pi i}{d}}\right)$ for all positive integers $d, n$ such that $d \mid n$.

Phrased in a different manner, the family is Lyndon-like if and only if the number of elements in $X_{n}$ fixed by $g^{d}$ is in bijection with $X_{d}$ where $g$ is an element of order $n$ in $C_{n}$. We note that the notion of Lyndon-like is also studied from a different perspective (called $q$-Gauß congruences) in [19].

EXAmple 2.10 (See [35, Prop. 4.4]). Let $W_{n k}$ be the set of words of length $n$ in the alphabet $[k]$. Let $C_{n}$ act on $W_{n k}$ by cyclic rotation. Take $f_{n}(q)=\sum_{w \in W_{n k}} q^{\operatorname{maj}(w)}$, where $\operatorname{maj}(w)$ is the sum over all indices $j$ such that $w_{j}>w_{j+1}$. Then $\left(W_{n k}, C_{n}, f_{n}(q)\right)$ exhibits the cyclic sieving phenomenon. Furthermore, if we fix $k$, this family of CSPtriples is Lyndon-like.

One can show that the group action on a Lyndon-like family $X_{n}$ corresponds to rotation on some set of words of length $n$, see [3, Prop. 34]. When $X_{n}$ is the set of binary words of length $n$, the orbits of length $n$ are in bijection with Lyndon words, see A001037 in [44]. Each Lyndon-like family of combinatorial objects then has an analogue of Lyndon words.

2.6. Burge WORDS AND RSK. The Robinson-Schenstedt-Knuth correspondence (RSK) is a famous combinatorial bijection with many different applications [27, 45]. The version we use in this paper is a bijection between pairs of certain biwords and pairs of semistandard Young tableaux. We note that the biwords we consider are not lexigraphically ordered, which is otherwise typical.

Definition 2.11. A Burge word is a two-line array with positive integers

$$
W=\left(\begin{array}{cccc}
i_{1} & i_{2} & \cdots & i_{m} \\
j_{1} & j_{2} & \cdots & j_{m}
\end{array}\right)
$$

sorted primarily increasingly in the first row and secondarily on the second row de-

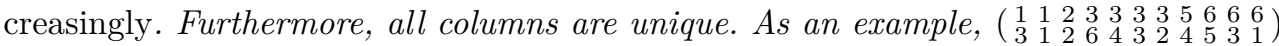
is a Burge word. A pair $\left(i_{c}, j_{c}\right)$ is called a biletter. The first row of $W$ is called the recording word and the second row of the biword is called the charge word - the reason for this terminology will be apparent in Proposition 5.5.

We use the same row insertion bumping algorithm as the standard RSK on biwords, which we assume the readers are familiar with. Our version of RSK and relevant properties is the third variant described by C. Krattenthaler [27, § 4.3].

Proposition 2.12. The RSK-algorithm yields a bijection between Burge words and pairs of fillings $(P, Q)$ of the same shape such that the insertion tableau $P$ is semistandard and the recording tableau $Q$ has the property that $Q^{t}$ is semistandard. 


\begin{tabular}{|c|c|c|c|c|c|c|c|c|}
\hline Inserted biletter & $\left(\begin{array}{l}1 \\
4\end{array}\right)$ & $\left(\begin{array}{l}1 \\
1\end{array}\right)$ & $\left(\begin{array}{l}2 \\
3\end{array}\right)$ & $\left(\begin{array}{l}2 \\
2\end{array}\right)$ & $\left(\begin{array}{l}4 \\
5\end{array}\right)$ & $\left(\begin{array}{l}5 \\
4\end{array}\right)$ & $\left(\begin{array}{l}5 \\
3\end{array}\right)$ & $\left(\begin{array}{l}5 \\
1\end{array}\right)$ \\
\hline$P$ & 4 & \begin{tabular}{|l|}
1 \\
4 \\
\end{tabular} & \begin{tabular}{|l|l|}
1 & 3 \\
4 & \\
\end{tabular} & \begin{tabular}{|l|l}
1 & 2 \\
3 & \\
\cline { 1 - 1 } 4 & \\
\cline { 1 - 1 } &
\end{tabular} & \begin{tabular}{|l|l|l}
1 & 2 & 5 \\
\cline { 1 - 1 } 3 & \multicolumn{3}{|l}{} \\
\cline { 1 - 1 } 4 & \multicolumn{1}{|l}{} \\
\end{tabular} & \begin{tabular}{|l|l|l}
1 & 2 & 4 \\
3 & 5 & \multicolumn{1}{|c}{} \\
\cline { 1 - 2 } 4 & \multicolumn{2}{|c}{} \\
\end{tabular} & \begin{tabular}{|l|l|l|}
1 & 2 & 3 \\
3 & 4 & \\
4 & 5 & \\
\end{tabular} & \begin{tabular}{|l|l|l}
1 & 1 & 3 \\
2 & 4 & \multicolumn{1}{|l}{} \\
3 & 5 & \\
\cline { 1 - 2 } 4 & \multicolumn{2}{|c}{} \\
\cline { 1 - 1 }
\end{tabular} \\
\hline$Q$ & 1 & \begin{tabular}{|l|}
1 \\
1 \\
\end{tabular} & \begin{tabular}{|l|l|}
1 & 2 \\
1 & \\
\end{tabular} & \begin{tabular}{|l|l}
1 & 2 \\
1 & \\
\cline { 1 - 1 } 2 & \\
\cline { 1 - 1 } &
\end{tabular} & \begin{tabular}{|l|l|l}
1 & 2 & 4 \\
1 & \multicolumn{3}{|l}{} \\
\cline { 1 - 1 } 2 & \multicolumn{1}{|l}{} \\
\end{tabular} & \begin{tabular}{|l|l|l}
1 & 2 & 4 \\
1 & 5 & \\
2 & \multicolumn{1}{|c}{} \\
\cline { 1 - 1 } &
\end{tabular} & \begin{tabular}{|l|l|l}
1 & 2 & 4 \\
1 & 5 & \\
2 & 5 & \multicolumn{1}{|c}{} \\
\end{tabular} & \begin{tabular}{|l|l|l|}
1 & 2 & 4 \\
1 & 5 & \\
5 & 5 & \\
5 & &
\end{tabular} \\
\hline
\end{tabular}

TABLE 2. Computing the image of a Burge word under RSK via a sequence of row insertions.

As an example of Proposition 2.12, the procedure in Table 2 shows that we have the following correspondence.

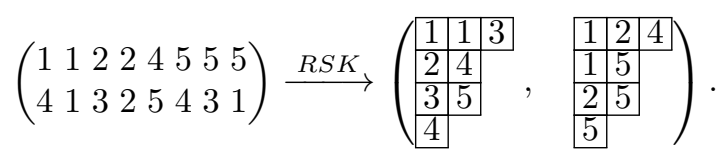

2.7. Symmetric functions And Plethysm. We use standard notation (see e.g. [32, 45]) for symmetric functions. We have the elementary symmetric functions $\mathrm{e}_{\lambda}$, complete homogeneous symmetric functions $h_{\lambda}$, the power-sum symmetric functions $p_{\lambda}$ and the Schur functions $\mathrm{s}_{\lambda}$. Recall also the standard involution on symmetric functions $\omega$, with the defining properties that for $\lambda \vdash n$,

$$
\omega\left(\mathrm{h}_{\lambda}\right)=\mathrm{e}_{\lambda}, \quad \omega\left(\mathrm{s}_{\lambda}\right)=\mathrm{s}_{\lambda^{\prime}}, \quad \omega\left(\mathrm{p}_{\lambda}\right)=(-1)^{n-\ell(\lambda)} \mathrm{p}_{\lambda} .
$$

We shall also require a few identities related to plethysm - for a comprehensive background on plethysm and the notation used, see J. Haglund's book [20]. In this paper, we only need the following few properties. When $f$ is a symmetric function, we let the plethystic substitution $\mathrm{p}_{k}[f]$ for $k \in \mathbb{N}$ be defined as

$$
\mathrm{p}_{k}[f]:=f\left(x_{1}^{k}, x_{2}^{k}, x_{3}^{k}, \ldots\right) .
$$

Note that in particular, $\mathrm{p}_{k}\left[\mathrm{p}_{m}\right]=\mathrm{p}_{k m}$. It is clear from the definition that for symmetric functions $f$ and $g$,

$$
\mathrm{p}_{k}[f+g]=\mathrm{p}_{k}[f]+\mathrm{p}_{k}[g] \text { and } \mathrm{p}_{k}[f \cdot g]=\mathrm{p}_{k}[f] \cdot \mathrm{p}_{k}[g] .
$$

LEMma 2.13. For any homogeneous symmetric function $f$ of degree $n$, we have that

$$
\mathrm{p}_{k}[\omega f]=(-1)^{(k+1) n} \omega\left(\mathrm{p}_{k}[f]\right) .
$$

Proof. Since plethysm is linear, it suffices to prove the identity for $f=\mathrm{p}_{\lambda}$, where $\lambda \vdash n$. We have that $\mathrm{p}_{k}\left[\omega \mathrm{p}_{\lambda}\right]$ is equal to $\mathrm{p}_{k}\left[(-1)^{n-\ell(\lambda)} \mathrm{p}_{\lambda}\right]=(-1)^{n-\ell(\lambda)} \mathrm{p}_{k \lambda}=$ $(-1)^{(n-\ell(\lambda))+(k n-\ell(\lambda))} \omega\left(\mathrm{p}_{k \lambda}\right)$ which can be simplified to $(-1)^{(k+1) n} \omega\left(\mathrm{p}_{k}\left[\mathrm{p}_{\lambda}\right]\right)$.

2.8. Hall-Littlewood AND NON-Symmetric Macdonald polynomials. The family of non-symmetric Macdonald polynomials, $\left\{\mathrm{E}_{\alpha}(\mathbf{x} ; q, t)\right\}_{\alpha}$ where $\alpha \in \mathbb{N}^{n}$ is a basis for $\mathbb{C}(q, t)\left[x_{1}, \ldots, x_{n}\right]$. These were introduced by E. Opdam [32, 34], and further developed by I. Cherednik [16]. The first definition of non-symmetric Macdonald polynomials is quite cumbersome and indirect. J. Haglund, M. Haiman and $\mathrm{N}$. Loehr [22] found a combinatorial formula for computing $\mathrm{E}_{\alpha}(\mathbf{x} ; q, t)$, using the notion of non-attacking fillings, thus generalizing F. Knop and S. Sahi's earlier formula 
for Jack polynomials [26]. In this paper, we shall only study a special case of the non-symmetric Macdonald polynomials, namely the case when $\lambda$ is a partition and $t=0$. Here, we use the same notation as P. Alexandersson and M. Sawney [6, 7], which differs slightly from Haglund et al. [22]. The notation $\mathrm{E}_{\alpha}(\mathbf{x} ; q, t)$ in this paper is equal to $E_{\operatorname{rev}(\alpha)}(\mathbf{x} ; q, t)$ in theirs where the composition has been reversed. Since we shall only study the specialization $\mathrm{E}_{\lambda}(\mathbf{x} ; q, 0)$, we do not introduce the non-symmetric Macdonald polynomials in full generality.

Let $\lambda=\left(\lambda_{1}, \ldots, \lambda_{\ell}\right)$ be a Young diagram, $m \in \mathbb{N}$ with $m \geqslant r$. Let $F: \lambda \rightarrow[m]$ be a filling of $\lambda$. Three boxes $a, b, c$ in $F$ form a triple if $a$ is just to the left of $b$ and $c$ in a row lower than $b$ and in the same column as $b$. The entries in a triple form an inversion-triple if they are ordered increasingly in a counter-clockwise orientation. If two entries in the triple are equal, then the entry with the largest subscript in (9) is considered to be the biggest.

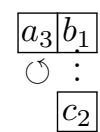

A filling of shape $\lambda$ is called a coinversion-free filling if every triple is an inversiontriple and the first column is strictly decreasing from top to bottom. The set of such fillings where the entries are in $[m]$ is denoted $\operatorname{COF}(\lambda, m)$, see Figure 2 for an example. Note that the conditions imply that every column in a coinversion-free filling must have distinct entries.

REMARK 2.14. The definition of coinversion-free filling is essentially the same as used by P. Alexandersson and M. Sawhney [6] and by J. Uhlin [48] with the exception that the aforementioned texts also include basements. However, it is easy to see that these different definitions both yield $\mathrm{E}_{\lambda}(\mathbf{x} ; q, 0)$. Arguably, our definition makes the results in this article more natural. S. Assaf [8] and S. Assaf, N. Gonzáles [9] study a generalized form of coinversion-free fillings, which also allows composition-shaped fillings. Therein, they are called semistandard key tabloids.

A descent ${ }^{(2)}$ of a filling $F$ is a box $(i, j)$ such that $F(i, j-1)<F(i, j)$. In particular, there are no descents in the first column. The set of descents is denoted $\operatorname{Des}(F)$. The leg of a box $b$ is the number of boxes that lie strictly to the right of $b$ in the diagram. In other words, if $b=(i, j)$, then $\operatorname{leg}(b)=\lambda_{i}-j$. The major index of $F$ is defined as

$$
\left.\operatorname{maj}(F)=\sum_{b \in \operatorname{Des}(F)}(\operatorname{leg}(b)+1)\right) .
$$

Given a filling $F$ of shape $\lambda$, we let the weight of $F, \operatorname{wt}(F)=\left(w_{1}, \ldots, w_{m}\right)$, be the vector such that $w_{i}$ counts the number of occurrences of $i$ in $F$. Furthermore, we let $\mathbf{x}^{F}$ denote the monomial $\prod_{(i, j) \in \lambda} x_{F(i, j)}$, see Figure 2 .

The (specialized) non-symmetric Macdonald polynomial $\mathrm{E}_{\lambda}(\mathbf{x} ; q, 0)$ is then defined as

$$
\mathrm{E}_{\lambda}\left(x_{1}, \ldots, x_{m} ; q, 0\right)=\sum_{F \in \operatorname{COF}(\lambda, m)} q^{\operatorname{maj}(F)} \mathbf{x}^{F} .
$$

One can verify that this definition agrees with the one given in [2]. Despite the name, the specialization $\mathrm{E}_{\lambda}(\mathbf{x} ; q, 0)$ is in fact a symmetric polynomial ${ }^{(3)}$ as we shall see below.

\footnotetext{
(2) Note that this seems non-standard compared to descents in words. This terminology is due to the usage of skyline diagrams used when describing the non-symmetric Macdonald polynomials [22]. We use English notation rather than skyline diagrams.

${ }^{(3)}$ There is an extension of the notion of inversion triples to diagrams indexed by weak compositions and then one has that $\mathrm{E}_{\lambda}(\mathbf{x} ; q, 0)$ is independent of the order of entries in $\lambda$, see [2, Prop. 17].
} 
Figure 2. A coinversion-free filling with descents marked and weight $(1,3,2,4,3,1,4)$. Its major index is $1+2+2+1=6$.

EXAMPLE 2.15. As can be computed by summing all monomials in Figure 3, we have that $\mathrm{E}_{(2,1)}\left(x_{1}, x_{2}, x_{3} ; q, 0\right)$ is given by

$$
\begin{aligned}
x_{1}^{2} x_{2}+x_{1} x_{2}^{2}+x_{1}^{2} x_{3}+x_{1} x_{3}^{2}+x_{2}^{2} x_{3}+ & x_{2} x_{3}^{2}+2 x_{1} x_{2} x_{3}+q x_{1} x_{2} x_{3} \\
& =(2+q) \mathrm{m}_{111}\left(x_{1}, x_{2}, x_{3}\right)+\mathrm{m}_{21}\left(x_{1}, x_{2}, x_{3}\right) .
\end{aligned}
$$

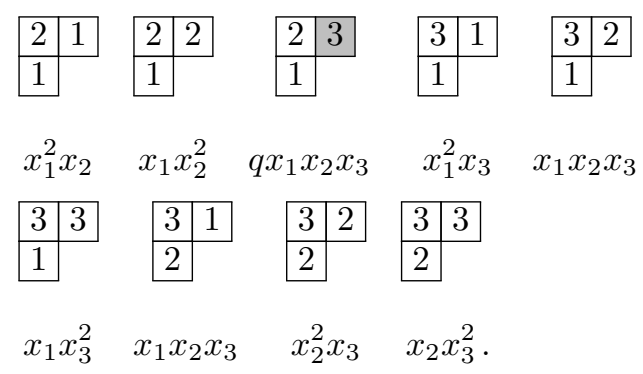

FiguRE 3. All coinversion-free fillings of shape $\lambda=(2,1)$ in three variables with their respective contribution to Equation (10).

We shall also briefly make use of the modified Macdonald polynomials further down. Let $\lambda / \mu$ be a skew shape and let $F: \lambda / \mu \rightarrow[m]$ be a filling with no restrictions. The notion of inversion triples with the cases in (9) is extended to skew shapes, where the box a may now lie outside the diagram.

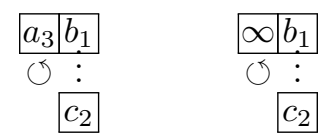

Such boxes outside the diagram $\lambda / \mu$ are considered to have value $\infty$, in which case it is required $F\left(b_{1}\right)>F\left(c_{2}\right)$ in order for the triple to be an inversion triple. Let $\operatorname{inv}(F)$ denote the total number of inversion triples in $F$. The notion of descent is extended to skew shapes, so that $(i, j)$ is a descent of $F$ if $F(i, j-1)<F(i, j)$, and we let $\operatorname{maj}(F)=\sum_{b \in \operatorname{Des}(F)}(\operatorname{leg}(b)+1)$ as before. Similarly, the notion of weight is extended in the natural way. The (skew) modified Macdonald polynomial $\tilde{\mathrm{H}}_{\lambda / \mu}(\mathbf{x} ; q, t)$ is defined as

$$
\tilde{\mathrm{H}}_{\lambda / \mu}\left(x_{1}, \ldots, x_{m} ; q, t\right)=\sum_{F: \lambda / \mu \rightarrow[m]} q^{\operatorname{maj}(F)} t^{\operatorname{inv}(F)} \mathbf{x}^{F} .
$$

This stabilizes to a symmetric function as $m \rightarrow \infty$. Note that $\left[t^{*}\right] \tilde{\mathrm{H}}_{\lambda}(\mathbf{x} ; q, t)=$ $\mathrm{E}_{\lambda}(\mathbf{x} ; q, 0)$, that is, the coefficient of the highest power of $t$ that appears is given by a specialized Macdonald polynomial. 
A lot of research has been devoted to study the family of modified Macdonald polynomials. The starting point for the formula in (12) is the reference [21], while the skew extension is the main topic in [10]

We shall also make use of the transformed Hall-Littlewood polynomials, $\mathrm{Q}_{\mu}^{\prime}(\mathbf{x} ; q)$. There are many different ways to define these, for example via the Kostka-Foulkes polynomials $K_{\lambda \mu}(q)$ :

$$
\mathrm{Q}_{\mu}^{\prime}(\mathbf{x} ; q)=\sum_{\lambda} K_{\lambda \mu}(q) \mathrm{s}_{\lambda}(\mathbf{x})
$$

We refer to $[17,32,47]$ for more background and properties. For completeness, we provide a combinatorial method for computing $K_{\lambda \mu}(q)$ in Section 2.4. The transformed Hall-Littlewood polynomials are closely related to our specialization of the non-symmetric Macdonald polynomials.

Proposition 2.16 (See [6, Thm. 14] or [20]). For any partition $\lambda$

$$
\mathrm{E}_{\lambda}(\mathbf{x} ; q ; 0)=\omega \mathrm{Q}_{\lambda^{\prime}}^{\prime}(\mathbf{x} ; q) \text {. }
$$

2.9. Evaluations at roots of Unity. Our first goal is to evaluate $\mathrm{E}_{\lambda}(\mathbf{x} ; q ; 0)$ when $q$ is a root of unity. We need a few intermediate results.

Proposition 2.17 (See [29, Thm. 2.1]). Let $\lambda$ be a partition of $n$. Let d be a positive integer and write $m_{j}(\lambda)=d m_{j}^{\prime}+r_{j}$ with $0 \leqslant r_{j}<d$. Furthermore, let $\xi$ be a primitive $d^{\text {th }}$ root of unity. Then

$$
\mathrm{Q}_{\lambda}^{\prime}(\mathbf{x} ; \xi)=\mathrm{Q}_{\tilde{\lambda}}^{\prime}(\mathbf{x} ; \xi) \prod_{j=1}^{n}\left(\mathrm{Q}_{\left(j^{d}\right)}^{\prime}(\mathbf{x} ; \xi)\right)^{m_{j}^{\prime}}
$$

where $\tilde{\lambda}$ is the partition $\left(n^{r_{n}}, \ldots, 2^{r_{2}}, 1^{r_{1}}\right)$.

Proposition 2.18 (See [29, Thm. 2.2]). Let $\xi$ be a primitive $n^{\text {th }}$ root of unity. Then

$$
\mathrm{Q}_{k^{n}}^{\prime}(\mathbf{x} ; \xi)=(-1)^{k(n-1)} \mathrm{p}_{n}\left[\mathrm{~h}_{k}(\mathbf{x})\right] \text {. }
$$

TheOREM 2.19. Let $\mu$ be an integer partition and $n \in \mathbb{N}$. Furthermore, let $\xi$ be a primitive $n^{\text {th }}$ root of unity and $d$ a divisor of $n$. Then

$$
\mathrm{E}_{n \mu}\left(1^{m} ; \xi^{d}, 0\right)=\prod_{j \geqslant 1}\left(\begin{array}{c}
m \\
j
\end{array}\right)^{d \cdot m_{j}\left(\mu^{\prime}\right)}
$$

Proof. We have that $\xi^{d}$ is a primitive $\left(\frac{n}{d}\right)^{\text {th }}$ root of unity. Since $m_{j}\left((n \mu)^{\prime}\right)=n \cdot m_{j}\left(\mu^{\prime}\right)$ we have by Proposition 2.17,

$$
\mathrm{Q}_{(n \mu)^{\prime}}^{\prime}\left(\mathbf{x} ; \xi^{d}\right)=\prod_{j \geqslant 1}\left(\mathrm{Q}_{j^{n / d}}^{\prime}\left(\mathbf{x} ; \xi^{d}\right)\right)^{d \cdot m_{j}\left(\mu^{\prime}\right)} .
$$

Moreover, Proposition 2.18 then gives that

$$
\mathrm{Q}_{(n \mu)^{\prime}}^{\prime}\left(\mathbf{x} ; \xi^{d}\right)=\prod_{j \geqslant 1}\left((-1)^{j(n / d-1)} \mathrm{p}_{n / d}\left[\mathrm{~h}_{j}(\mathbf{x})\right]\right)^{d \cdot m_{j}\left(\mu^{\prime}\right)} .
$$

Applying the $\omega$-involution on both sides of (17) and using Proposition 2.16 and Lemma 2.13 gives

$$
\mathrm{E}_{n \mu}\left(\mathbf{x} ; \xi^{d}, 0\right)=\prod_{j \geqslant 1}\left(\mathrm{p}_{n / d}\left[\mathrm{e}_{j}(\mathbf{x})\right]\right)^{d \cdot m_{j}\left(\mu^{\prime}\right)}=\prod_{j \geqslant 1}\left(\mathrm{e}_{j}\left(x_{1}^{\frac{n}{d}}, x_{2}^{\frac{n}{d}}, \ldots\right)\right)^{d \cdot m_{j}\left(\mu^{\prime}\right)} .
$$


Finally, we set $\left(x_{1}, \ldots, x_{m}\right)=\left(1, q, q^{2}, \ldots, q^{m-1}\right)$ and remaining variables are set to zero. Formulas for the principal specialization of elementary symmetric functions, see [45, p. 303], give

$$
\mathrm{E}_{n \mu}\left(1, q, q^{2}, \ldots, q^{m-1} ; \xi^{d}, 0\right)=\prod_{j \geqslant 1}\left(q^{\frac{n}{d}\left(\begin{array}{c}
j \\
2
\end{array}\right)}\left[\begin{array}{c}
m \\
j
\end{array}\right]_{q^{\frac{n}{d}}}\right)^{d \cdot m_{j}\left(\mu^{\prime}\right)} .
$$

In particular, with $q=1$ we obtain (16).

EXAMPLE 2.20. Let $\mu=885322, n=12$ and $d=4$. Then $\mu^{\prime}=66433222$ and

$$
\mathrm{E}_{12 \mu}\left(1^{m} ; e^{\frac{2 \pi i 4}{12}}, 0\right)=\left(\left(\begin{array}{c}
m \\
2
\end{array}\right)^{3}\left(\begin{array}{c}
m \\
3
\end{array}\right)^{2}\left(\begin{array}{c}
m \\
4
\end{array}\right)\left(\begin{array}{c}
m \\
6
\end{array}\right)^{2}\right)^{4}
$$

\section{CyCliC Sieving ON COINVERSION-FreE FILlings}

Recall that $\operatorname{COF}(n \lambda, m)$ denotes the set of coinversion-free fillings of shape $n \lambda$ and entries in $[m]$. In order to define the cyclic group action on $\operatorname{COF}(n \lambda, m)$, we need to keep the following result in mind.

Proposition 3.1 (See $[6,7]$ ). Let $\lambda$ be a partition with $\ell$ parts and let $S_{1}, S_{2}, \ldots, S_{\ell}$ be subsets of $[\mathrm{m}]$ such that $\left|S_{j}\right|=\lambda_{j}$. Then there is a unique coinversion-free filling $F$ of shape $\lambda^{\prime}$ such that the entries in column $j$ of $F$ are given by $S_{j}$.

Note that this proposition implies that $\mathrm{E}_{\lambda^{\prime}}(\mathbf{x} ; 1 ; 0)=\mathrm{e}_{\lambda}(\mathbf{x})$, and this identity generalizes to the non-symmetric setting, see [7].

Let $\phi$ act on $\operatorname{COF}(n \lambda, m)$ by cyclically shifting the first $n$ columns one step to the right, the next $n$ columns one step to the right, and so on. Finally, the elements in each column are rearranged so that the result is again a coinversion-free filling in $\operatorname{COF}(n \lambda, m)$. This action is well-defined according to Proposition 3.1. Clearly, $\phi$ generates a cyclic group of order $n$ acting on $\operatorname{COF}(n \lambda, m)$, see Figure 4 for an example. We are now ready to prove one of the main results of the paper.

$$
\begin{array}{|l|l|l|l|l|l|}
\hline 6 & 4 & 2 & 1 & 1 & 6 \\
\hline 5 & 2 & 6 & 3 & 4 & 3 \\
\hline 2 & 1 & 4 &
\end{array} \quad \stackrel{\phi}{\rightarrow} \quad \begin{array}{|l|l|l|l|l|l|l|}
6 & 6 & 4 & 3 & 3 & 1 \\
\hline 4 & 2 & 2 & 6 & 1 & 4 \\
\hline 2 & 5 & 1 & \multicolumn{1}{|l}{} \\
\cline { 1 - 3 } &
\end{array}
$$

FIGURE 4. The action of $\phi$ on a filling of shape $n \lambda=3(2,2,1)$.

ThEOREM 3.2. For every integer partition $\lambda$ and $n \geqslant 1$, the triple

$$
\left(\operatorname{COF}(n \lambda, m),\langle\phi\rangle, \mathrm{E}_{n \lambda}\left(1^{m} ; q, 0\right)\right)
$$

exhibits the cyclic sieving phenomenon. Moreover, the family

$$
\left\{\left(\operatorname{COF}(n \lambda, m),\langle\phi\rangle, \mathrm{E}_{n \lambda}\left(1^{m} ; q, 0\right)\right)\right\}_{n=1}^{\infty}
$$

is a Lyndon-like family.

Proof. We first need to compute the number of elements fixed under $\phi^{d}$ whenever $d \mid n$. Since coinversion-free fillings are uniquely determined by their column sets, a coinversion-free filling in $\operatorname{COF}(n \lambda, m)$ fixed under $\phi^{d}$ is uniquely determined by the first $d$ columns in each consecutive block of $n$ columns. Hence,

$$
\#\left\{F \in \operatorname{COF}(n \lambda, m): \phi^{d} \cdot F=F\right\}=\# \operatorname{COF}(d \lambda, m)=\prod_{j \geqslant 1}\left(\begin{array}{c}
m \\
j
\end{array}\right)^{d \cdot m_{j}\left(\lambda^{\prime}\right)} .
$$

By using Theorem 2.19, the statements in the theorem now follows. 
There is another natural group action on coinversion-free fillings. Pick $\sigma \in S_{m}$ and let $\sigma: \operatorname{COF}(\lambda, m) \rightarrow \operatorname{COF}(\lambda, m)$ act by letting $F^{\prime}=\sigma(F)$ be given by $F^{\prime}(i, j)=$ $\sigma F(i, j)$ for all $(i, j) \in \lambda$, followed by rearranging the elements in each column of $F^{\prime}$ to obtain a new coinversion-free filling. By Proposition 3.1, this action is well defined. Let $C_{M}=\langle\sigma\rangle$. Recall the notion of $C_{M}$ acting nearly freely on $[m]$ from Example 2.8. This induces a $C_{M}$-action on $\operatorname{COF}(\lambda, m)$.

TheOREM 3.3. Let $n$ and $m$ be positive integers and let $\lambda$ be a partition. If $C_{n}$ acts nearly freely on $[m]$, then

$$
\left(\operatorname{COF}(\lambda, m), C_{n}, \mathrm{E}_{\lambda}\left(1, q, q^{2}, \ldots, q^{m-1} ; 1,0\right)\right)
$$

exhibits the cyclic sieving phenomenon.

Proof. The proof of the case when $\lambda$ is rectangular can be found in [48], and extends to the general case without extra effort. Suppose $C_{n}$ is generated by $\sigma$. Write $\lambda^{\prime}=\left(\lambda_{1}^{\prime}, \ldots, \lambda_{c}^{\prime}\right)$ and consider the set of $c$-tuples $\mathbf{s}=\left(s_{1}, \ldots, s_{c}\right)$, so that $s_{i} \subseteq[m]$ and $\# s_{i}=\lambda_{i}^{\prime}$. We let $C_{n}$ act on such $c$-tuples by letting $\sigma \mathbf{s}=\left(\sigma s_{1}, \ldots, \sigma s_{c}\right)$. Proposition 3.1 implies that we can identify a coinversion-free filling with its column sets. Hence, there is a bijection from $\operatorname{COF}(\lambda, m)$ to the set of $c$-tuples on the above form and it is clear that this bijection is equivariant with respect to $\sigma$, so it suffices to show that the set of $c$-tuples exhibits the cyclic sieving phenomenon.

But the set of $c$-tuples is a direct product of sets which we know exhbit the cyclic sieving phenomenon - this is just Example 2.8. Furthermore, the product of the CSPpolynomials in the example agree with (18) (when $\xi=1$ ). It is straightforward to show that that CSP is preserved under taking direct products (see e.g. [12, Prop. 2.2], [1, Rem. 2.3] or [48, Lem. $4.13(i)])$ so we are done.

EXAMPLE 3.4. Note that the above theorem does in general not hold if $C_{M}$ does not act nearly freely on $[m]$. Suppose that $n=k=1$ and $C_{4}$ is generated by the permutation $\langle(1234)\rangle$. Then, the CSP-polynomial $\mathrm{E}_{n^{k}}\left(1, q, q^{2}, \ldots, q^{m-1} ; 1,0\right)=1+$ $q+q^{2}+q^{3}+q^{4}+q^{5}$, which evaluated at a primitive $4^{\text {th }}$ of unity $\xi=i$ yields $1+i$.

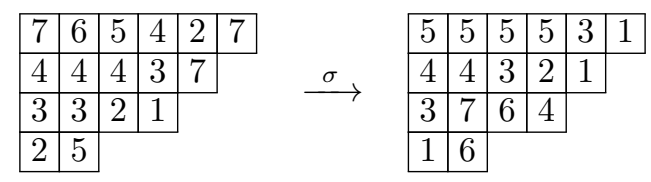

Figure 5. An example of the action of $\sigma=(1234567)$.

\section{Refined CSP on STRETCHED SPECIALIZED MACDONALD FiLlings}

After the project started, we realized that Theorem 3.2 is very much connected with [37]. In fact, the main result of B. Rhoades implies a refined version of Theorem 3.2 as we shall see later in this section. Note however that the connection between his set of combinatorial objects (01-matrices), and coinversion-free fillings and Macdonald polynomials passes through RSK, which in our opinion makes it worthwhile to give the direct proof Theorem 3.2.

Let $\left[\mathrm{m}_{\nu}\right] f$ denote the coefficient of $\mathrm{m}_{\nu}$ in the symmetric function $f$. It follows from Proposition 2.16 and (13) that

$$
\left[\mathrm{m}_{\nu}\right] \mathrm{E}_{\lambda}(\mathbf{x} ; q, 0)=\sum_{\mu} K_{\mu \nu}(1) K_{\mu^{\prime} \lambda^{\prime}}(q) .
$$


THEOREM 4.1 (See [37, Thm. 1.4]). Let $\mu$ and $\nu$ be compositions of $n$, with cyclic symmetries $a$ and $b$, respectively. Let $X(\mu, \nu)$ be the set of $\ell(\lambda) \times \ell(\nu)$ binary matrices with row content $\mu$ and column-content $\nu$. Then the product $C_{\ell(\mu) / a} \times C_{\ell(\nu) / b}$ act on $X$ by a-fold row-rotation and b-fold column-rotation, respectively. Then

$$
\left(X(\mu, \nu), C_{\ell(\mu) / a} \times C_{\ell(\nu) / b}, \delta_{n}(q, t) \sum_{\lambda \vdash n} K_{\lambda \mu}(q) K_{\lambda^{\prime} \nu}(t)\right)
$$

exhibits the bi-cyclic sieving phenomenon. Here, $\delta_{n}(q, t)$ is a messy polynomial taking on values \pm 1 at relevant roots of unity. Furthermore, one can check that $\delta_{n}(q, t) \equiv 1$ in the case $q=1$.

By only considering the action on the columns in Theorem 4.1, we get the following corollary.

Corollary 4.2. Let $n \in \mathbb{N}, \lambda \vdash k$ where $\ell=\ell(\lambda), \nu \vDash n k$ with $m$ parts and let $X(\nu, n \lambda)$ be the set of $m \times n \lambda_{1}$ binary matrices with row-content $\nu$ and column-content given by the conjugate of $n \lambda$. Let $C_{n}$ act on $X(\nu, n \lambda)$ by cyclic rotation of each block of $n$ consecutive columns. Then

$$
\left(X(\nu, n \lambda), C_{n}, \sum_{\mu \vdash n k} K_{\mu, \nu}(1) K_{\mu^{\prime},(n \lambda)^{\prime}}(q)\right)
$$

is a CSP-triple.

Proof. Note that there is an easy correspondence between $n$-fold rotation of columns and rotation of each block of $n$ consecutive columns.

Recall the definition of $\phi$ in Section 3, which cyclically shifts each block of $n$ consecutive columns.

THEOREM 4.3. Let $n \in \mathbb{N}, \lambda \vdash k$ and let $\nu$ be a weak composition of $n k$ with $m$ parts. Then

$$
\left(\operatorname{COF}(n \lambda, \nu),\langle\phi\rangle,\left[\mathrm{m}_{\nu}\right] \mathrm{E}_{n \lambda}(\mathbf{x} ; q, 0)\right)
$$

exhibits the cyclic sieving phenomenon.

Proof. Let $\varphi$ denote the one-step cyclic rotation of each block of $n$ consecutive columns in a matrix. By $(20)$ it suffices to show that there is a bijection $A: X(\nu, n \lambda) \rightarrow$ $\operatorname{COF}(n \lambda, \nu)$ such that $\phi \circ A(M)=A \circ \varphi(M)$. We let $A(M)=F$ if and only if

$$
M(i, j)=1 \quad \Longleftrightarrow \quad \text { column } j \text { in } F \text { contains } i \text {. }
$$

It is straightforward to verify that $\phi \circ A(M)=A \circ \varphi(M)$, so fixed-points under $\varphi^{d}$ are mapped to fixed-points under $\phi^{d}$ for all $d \in \mathbb{Z}$. This proves the theorem.

See Figure 6 for an illustration of Theorem 4.3 .

REMARK 4.4. In an upcoming article [5], we prove a result reminiscent of Theorem 4.3. We show that there exists a cyclic group action $\rho$ of order $n$ such that $\left(\operatorname{SYT}(n \lambda),\langle\rho\rangle, f^{n \lambda}(q)\right)$ is a CSP-triple, where $f^{\lambda}(q):=K_{\lambda, 1^{n}}(q)$ is the major-index generating function on standard Young tableaux. We now have two families of fillings where stretched shapes admit CSP. Computer experiments on various other families of stretched symmetric functions suggest additional instances of CSP. This raises the question if there is some general theory behind these observations. 


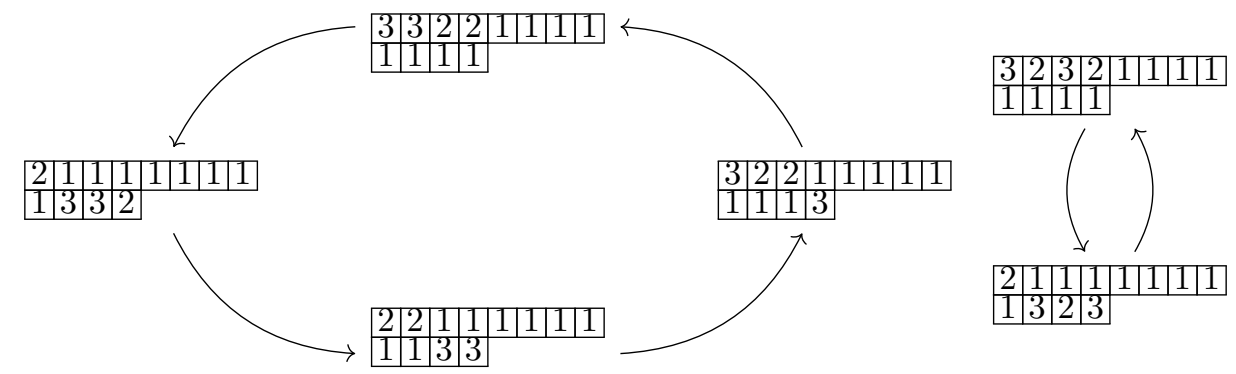

Figure 6. The orbits of $\operatorname{COF}(84,(8,2,2))$ under $\phi$. The CSPpolynomial is $f(q)=\left[\mathrm{m}_{(8,2,2)}\right] \mathrm{E}_{84}(\mathbf{x} ; q, 0)=1+q+q^{2}+q^{3}+q^{4}+q^{6}$. One can easily check that for a primitive $4^{\text {th }}$ root of unity $\xi, f(\xi)=0$, $f\left(\xi^{2}\right)=2$ and $f\left(\xi^{4}\right)=6$ and thus agreeing with the definition of CSP in $(7)$.

\section{Skew Specialized Macdonald polynomials}

There is a natural generalization of $\mathrm{E}_{\lambda}(\mathbf{x} ; q, 0)$ to skew diagrams. In this section, we shall see that $\mathrm{E}_{\lambda / \mu}(\mathbf{x} ; q, 0)$ is symmetric and Schur positive. Interestingly, the coefficients in the Schur expansion are not related to skew Kostka-Foulkes polynomials which at first glance is a natural guess.

A skew specialized Macdonald filling of shape $\lambda / \mu$ is a filling $F$ of the skew shape $\lambda / \mu$ such that each column of $F$ contains distinct entries, the first column is strictly decreasing, and every triple in $F$ is an inversion-triple as in $(11)$. We let $\operatorname{COF}(\lambda / \mu)$ denote the set of all such fillings. It is not difficult to see that Proposition 3.1 can be generalized to the skew-case as well. In other words a skew specialized Macdonald filling is completely determined by the shape of the diagram and the ordered tuple of column sets.

Definition 5.1. Let $\lambda / \mu$ be a skew shape, and define the skew (specialized) nonsymmetric Macdonald polynomial as

$$
\mathrm{E}_{\lambda / \mu}(\mathbf{x} ; q, 0):=\sum_{F \in \operatorname{COF}(\lambda / \mu)} \mathbf{x}^{F} q^{\operatorname{maj}(F)} .
$$

One can quite easily see that these polynomials generalize the skew Schur functions:

$$
\mathrm{E}_{\lambda / \mu}(\mathbf{x} ; 0,0)=\mathrm{s}_{\lambda / \mu}(\mathbf{x})
$$

As in the non-skew case, the functions $\mathrm{E}_{\lambda / \mu}(\mathbf{x} ; q, 0)$ are actually symmetric, and we are justified to work in any number of variables. It is clear from the definition that

$$
\mathrm{E}_{\lambda / \mu}(\mathbf{x} ; q, 0)=\left[t^{*}\right] \tilde{\mathrm{H}}_{\lambda / \mu}(\mathbf{x} ; q, t) .
$$

The fact that these are symmetric follows from Theorem 5.7 below. Symmetry was proved earlier in the non-skew case [48] by using a variant of the LascouxSchützenberger involutions, see Definition 6.5 below.

Given a filling $F \in \operatorname{COF}(\lambda / \mu)$ and some large integer $M$, we define the extended filling $\hat{F}$ as the filling of shape $\lambda$ obtained from $F$ as

$$
\hat{F}(i, j)= \begin{cases}M-i & \text { if }(i, j) \in \mu \\ F(i, j) & \text { otherwise. }\end{cases}
$$

Note that $\hat{F}$ is a specialized Macdonald filling and that $\operatorname{maj}(F)=\operatorname{maj}(\hat{F})$. We shall make use of this definition in the next subsection. 


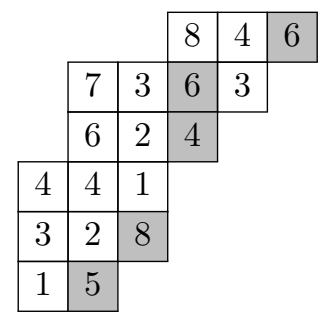

\begin{tabular}{|c|c|c|c|c|}
\hline$\hat{19}$ & $\hat{19}$ & $\hat{19}$ & 8 & 4 \\
\hline$\hat{18}$ & 7 & 3 & 6 & 3 \\
\hline$\hat{17}$ & 6 & 2 & 4 & \\
\hline 4 & 4 & 1 & & \\
\hline 3 & 2 & 8 & & \\
\hline 1 & 5 & & & \\
\hline
\end{tabular}

FiguRE 7. Left: A skew specialized Macdonald filling of shape $\lambda / \mu$ with $\lambda=(6,5,4,3,3,2)$ and $\mu=(3,1,1)$ with descents marked. The weight of the filling is $(2,2,3,4,1,3,1,2)$. Right: $\hat{F}$ with $M=20$.

5.1. Charge, RSK And Schur EXPAnsion. We assume that the reader is familiar with the (row-insertion) Robinson-Schenstedt-Knuth correspondence (RSK), described briefly in Section 2.6.

DEFINITION 5.2. Let $\mu$ be a partition and let $w$ be a word with content $\beta$ (which can be a weak composition) such that $\mu+\beta$ is a partition. The postfix charge charge $_{\mu}(w)$ is defined via the usual charge statistic as

$$
\operatorname{charge}_{\mu}(w):=\operatorname{charge}\left(w \cdot \ell^{\mu_{\ell}} \cdots 2^{\mu_{2}} 1^{\mu_{1}}\right) .
$$

That is, we concatenate a postfix to $w$ with content $\mu$, where the letters appear in decreasing order. For example, charge $_{21}(12231233)=\operatorname{charge}(12231233 \cdot 211)=2$.

Recall the definition of elementary Knuth transforms, stating that $y z x \sim_{K} y x z$ whenever $x<y \leqslant z$ and $x z y \sim_{K} z x y$ whenever $x \leqslant y<z$. Two words are Knuthequivalent, if one can obtain one from the other via a sequence of elementary Knuth transforms. If $w$ has partition-content, then its equivalence class contains a unique word which is the reading word of a semistandard Young tableau. Moreover, two words of partition content, $w$ and $w^{\prime}$ are Knuth-equivalent if and only if they insert to the same semistandard Young tableau under RSK, see [18, p. 22] or [15, Cor. 2.3.21].

LEMMA 5.3. Let $\mu$ be a partition and suppose $u$ and $v$ are Knuth-equivalent. Then $u$. $\ell^{\mu_{\ell}} \cdots 2^{\mu_{2}} 1^{\mu_{1}}$ and $v \cdot \ell^{\mu_{\ell}} \cdots 2^{\mu_{2}} 1^{\mu_{1}}$ are Knuth-equivalent and charge ${ }_{\mu}(u)=\operatorname{charge}_{\mu}(v)$.

Proof. The first statement follows easily from the definition of Knuth-equivalence. Furthermore, if two words are Knuth-equivalent, they have the same charge, [15, Cor. 2.4.38].

Recall the notion of a Burge word from Definition 2.11.

DeFinition 5.4. For each skew specialized Macdonald filling $F$ we associate a Burge word $W=W(F)$ as follows. For each entry $e=F(i, j)$, let $\left(\begin{array}{c}e \\ j\end{array}\right)$ be a biletter in $W$. Take $W$ to be the (unique) Burge word with all such biletters.

The non-skew case of Definition 5.4 was first given in [6]. Recall Proposition 3.1 and note that we can easily recover the column sets of $F$ from $W$. We send fillings in $\operatorname{COF}(\lambda / \mu, m)$ to biwords where the top row is a weakly increasing sequence with elements in $[m]$, and the bottom row has $j$ entries equal to $\lambda_{j}^{\prime}-\mu_{j}^{\prime}$ such that entries are strictly decreasing on each block of identical elements in the top row. This is a bijection when we fix some shape $\lambda / \mu$. However, two skew specialized Macdonald 
fillings of different shapes may give the same biword. For example,

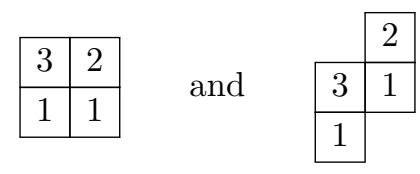

both have the same biword $\left(\begin{array}{llll}1 & 1 & 2 & 3 \\ 2 & 1 & 2 & 1\end{array}\right)$.

We let $\operatorname{ins}(F)$ and $\operatorname{rec}(F)$ denote the the insertion tableau and the recording tableau, respectively, after performing RSK on $W(F)$. Note that the content of $F$ is equal to the content of $P$ and is also given by the bottom row of $W$ while the content of $Q$ is given by the top row of $W$. Moreover, we let the charge word $\mathrm{cw}(F)$ denote the bottom row of $W$.

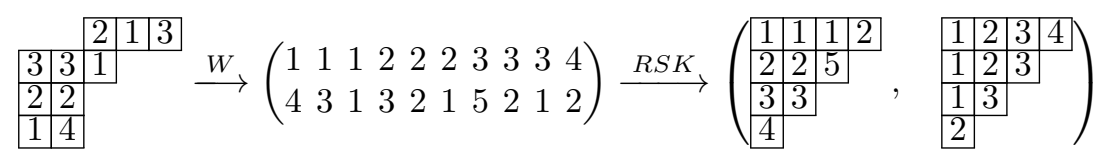

FIGURE 8. A skew specialized Macdonald filling $F$, the corresponding biword, and the result $(P, Q)=(\operatorname{ins}(F), \operatorname{rec}(F))$ under RSK.

Proposition 5.5. Suppose $\lambda / \mu$ is a skew shape and $F \in \operatorname{COF}(\lambda / \mu)$. Then $\operatorname{maj}(F)=$ charge $_{\mu^{\prime}}(\mathrm{cw}(F))$.

Proof. A short proof in the case $\mu=\varnothing$ was given in [6, Thm. 16]. Let $F \in \operatorname{COF}(\lambda / \mu)$ and let $\hat{F}$ be the extended filling of $F$ as in $(23)$. Recall that $\operatorname{maj}(F)=\operatorname{maj}(\hat{F})$. It follows from the definition of the biword that $\mathrm{cw}(\hat{F})=\mathrm{cw}(F) \cdot \ell^{\mu_{\ell}^{\prime}} \cdots 2^{\mu_{2}^{\prime}} 1^{\mu_{1}^{\prime}}$. The case $\mu=\varnothing \operatorname{implies}$ that $\operatorname{maj}(F)=\operatorname{maj}(\hat{F})=\operatorname{charge}(\operatorname{cw}(\hat{F}))=\operatorname{charge}_{\mu^{\prime}}(\operatorname{cw}(F))$.

Proposition 5.6. RSK provides a bijection

$$
\operatorname{COF}(\lambda / \mu) \stackrel{R S K}{\longrightarrow} \bigcup_{\nu \vdash|\lambda / \mu|} \operatorname{SSYT}(\nu, \alpha) \times \operatorname{SSYT}\left(\nu^{\prime}\right), \quad \alpha_{i}:=\lambda_{i}^{\prime}-\mu_{i}^{\prime},
$$

such that if $F \stackrel{R S K}{\longleftrightarrow}(P, Q)$ then $\operatorname{maj}(F)=\operatorname{charge}_{\mu^{\prime}}(\operatorname{cw}(F))=\operatorname{charge}_{\mu^{\prime}}(\operatorname{rw}(P))=$ $\operatorname{charge}_{\mu^{\prime}}(P)$.

Proof. The Robinson-Schenstedt-Knuth correspondence has the essential property that if the word $w$ inserts to $P$ under RSK, then charge $(w)=\operatorname{charge}(P)$. This is just a restatement of the fact that Knuth-equivalent words have the same charge. Using this property, Proposition 5.5 and Lemma 5.3, the statement follows.

We now have the setup needed to prove the following theorem.

Theorem 5.7 (The Schur expansion of skew specialized Macdonald polynomials). Let $\lambda / \mu$ be a skew shape and let $\alpha$ be the weak composition given by $\alpha_{i}:=\lambda_{i}^{\prime}-\mu_{i}^{\prime}$. Then

$$
\mathrm{E}_{\lambda / \mu}(\mathbf{x} ; q, 0):=\sum_{\nu \vdash|\lambda / \mu|} \mathrm{s}_{\nu^{\prime}}(\mathbf{x}) \sum_{T \in \operatorname{SSYT}(\nu, \alpha)} q^{\text {charge }_{\mu^{\prime}}(T)} .
$$

Proof. By definition, $\mathrm{E}_{\lambda / \mu}(\mathbf{x} ; q, 0)$ is equal to $\sum_{F \in \operatorname{COF}(\lambda / \mu)} \mathbf{x}^{F} q^{\operatorname{maj}(F)}$, which is equal to $\sum_{F \in \operatorname{COF}(\lambda / \mu)} \mathbf{x}^{F} q^{\text {charge }_{\mu^{\prime}}(\mathrm{cw}(F))}$ by using Proposition 5.5. Applying the RSK bijection in (24), we then have that

$$
\mathrm{E}_{\lambda / \mu}(\mathbf{x} ; q, 0)=\sum_{\nu} \mathrm{s}_{\nu^{\prime}}(\mathbf{x}) \sum_{P \in \operatorname{SSYT}(\nu, \alpha)} q^{\text {charge }_{\mu^{\prime}}(P)},
$$

which is exactly the statement in (25). 
Cyclic sieving, skew Macdonald polynomials and Schur positivity

\begin{tabular}{ll}
\hline Shape $\lambda / \mu$ & Schur expansion of $\mathrm{E}_{\lambda / \mu}(\mathbf{x} ; q, 0)$ \\
\hline 1 & $\mathrm{~s}_{1}$ \\
\hline 2 & $\mathrm{~s}_{2}+q \mathrm{~s}_{11}$ \\
11 & $\mathrm{~s}_{11}$ \\
$21 / 1$ & $\mathrm{~s}_{2}+\mathrm{s}_{11}$ \\
\hline 3 & $\mathrm{~s}_{3}+\left(q+q^{2}\right) \mathrm{s}_{21}+q^{3} \mathrm{~s}_{111}$ \\
21 & $\mathrm{~s}_{21}+q \mathrm{~s}_{111}$ \\
111 & $\mathrm{~s}_{111}$ \\
$22 / 1$ & $\mathrm{~s}_{21}+q \mathrm{~s}_{111}$ \\
$31 / 1$ & $\mathrm{~s}_{3}+(1+q) \mathrm{s}_{21}+q \mathrm{~s}_{111}$ \\
$211 / 1$ & $\mathrm{~s}_{21}+\mathrm{s}_{111}$ \\
$321 / 21$ & $\mathrm{~s}_{3}+2 \mathrm{~s}_{21}+\mathrm{s}_{111}$ \\
\hline
\end{tabular}

TABLE 3. Here are the Schur expansions of $\mathrm{E}_{\lambda / \mu}(\mathbf{x} ; q, 0)$ in the cases $|\lambda|-|\mu| \leqslant 3$.

Recall that the Littlewood-Richardson coefficients $c_{\mu \nu}^{\lambda}$ are defined via the relation $\mathrm{s}_{\mu} \mathrm{s}_{\nu}=\sum_{\lambda} c_{\mu \nu}^{\lambda} \mathrm{s}_{\lambda}$, and that the skew Schur functions expand as $\mathrm{s}_{\lambda / \mu}=\sum_{\nu} c_{\mu \nu}^{\lambda} \mathrm{s}_{\nu}$.

COROLlary 5.8 (A Littlewood-Richardson rule). Let $\lambda / \mu$ be a skew shape and let $\alpha$ be the weak composition $\alpha_{i}:=\lambda_{i}^{\prime}-\mu_{i}^{\prime}$. Let

$$
K_{\lambda / \mu}^{\nu}(q):=\sum_{T \in \operatorname{SSYT}(\nu, \alpha)} q^{{\text {charge } \mu^{\prime}}(T)} .
$$

\section{Then}

(a) $K_{\lambda / \mu}^{\nu}(0)=c_{\mu \nu}^{\lambda}$, a Littlewood-Richardson coefficient,

(b) $K_{\lambda / \mu}^{\nu}(1)=K_{\nu \alpha}$, a Kostka coefficient, and

(c) the product

$$
\begin{gathered}
\mathrm{E}_{\lambda}(\mathbf{x} ; q, 0) \mathrm{E}_{\mu}(\mathbf{x} ; q, 0)=\sum_{\nu} K_{\kappa / c^{r}}^{\nu}(q) \mathrm{s}_{\nu^{\prime}}(\mathbf{x}) \\
\text { where } c=\mu_{1}, r=\ell(\lambda) \text {, and } \kappa=\left(c+\lambda_{1}, \ldots, c+\lambda_{\ell(\lambda)}, \mu_{1}, \mu_{2}, \ldots, \mu_{\ell(\mu)}\right) .
\end{gathered}
$$

Proof. The first and second identity follow from Theorem 5.7 and (22) and using the fact that $c_{\mu \nu}^{\lambda}=c_{\mu^{\prime} \nu^{\prime}}^{\lambda^{\prime}}$. The third identity follows from the observation that the product in the left hand side can be realized as a single skew specialized Macdonald polynomial, $\mathrm{E}_{(\lambda+c, \mu) / c^{r}}(\mathbf{x} ; q, 0)$, see the diagram in $(27)$.

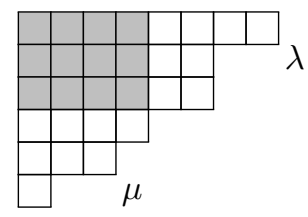

Note that the first property in the corollary implies that

$$
c_{\mu \nu}^{\lambda}=\left|\left\{T \in \operatorname{SSYT}(\nu, \lambda-\mu): \operatorname{charge}_{\mu}(T)=0\right\}\right|,
$$

where $\lambda-\mu$ denotes the integer vector where the $i^{\text {th }}$ entry is $\lambda_{i}-\mu_{i}$. This also follows from [24, Rem. 14 and Cor. 21], so this is not a new characterization of 
Littlewood-Richardson coefficients. We ask if there is a connection between the coefficients $K_{\lambda / \mu}^{\nu}(q)$ and the parabolic Kostka polynomials, whose constant terms are also Littlewood-Richardson coefficients, see [25, 43] for details.

We end this subsection with proving an additional property of postfix-charge. Recall that the charge statistic is Mahonian, meaning that $\sum_{\sigma \in S_{n}} q^{\text {charge }(\sigma)}=[n]_{q}$ ! . There is a natural generalization of this identity for $\operatorname{charge}_{\mu}(\cdot)$.

Proposition 5.9. Let $\mu$ be an integer partition and $n \geqslant 0$. Then

$$
\sum_{\sigma \in S_{n}} q^{\text {charge }_{\mu}(\sigma)}=n ! \prod_{i \geqslant 1} \frac{\left[\lambda_{i}-\mu_{i}\right]_{q} !}{\left(\lambda_{i}-\mu_{i}\right) !}
$$

where $\lambda^{\prime}=\left(\mu_{1}^{\prime}+1, \mu_{2}^{\prime}+1, \ldots, \mu_{n}^{\prime}+1, \mu_{n+1}^{\prime}, \ldots\right)$.

Proof. First note that the shape $\lambda / \mu$ has exactly one box in each of the first $n$ columns. For example, $\mu=53111$ and $n=8$ gives the following skew shape $\lambda / \mu$ :

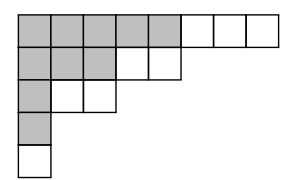

We use Proposition 5.5 together with the fact that every permutation appear as charge word appears exactly one when summing over fillings with weight $1^{n}$. Hence,

$$
\left[x_{1} x_{2} \cdots x_{n}\right] \mathrm{E}_{\lambda / \mu}(\mathbf{x} ; q)=\sum_{\sigma \in S_{n}} q^{\operatorname{charge}_{\mu}(\sigma)} .
$$

But the left hand side is easy to compute directly since the rows of $\lambda / \mu$ occupy disjoint columns; there are $n ! /\left(\left(\lambda_{1}-\mu_{1}\right) \cdots\left(\lambda_{\ell}-\mu_{\ell}\right)\right)$ ways to distribute $\{1,2, \ldots, n\}$ in the rows of $\lambda / \mu$. Furthermore, in row $i$, the major index gives the Mahonian distribution $\left[\lambda_{i}-\mu_{i}\right]_{q}$ ! when summing over all permutations of the entries. This implies the formula.

We remark that there is also the notion of skew Kostka-Foulkes polynomials, $K_{\lambda / \mu, \nu}(q)$ see [15]. They are defined as in (5), where the sum now ranges over the elements in $\operatorname{SSYT}(\lambda / \mu, \nu)$. To our knowledge, there is no obvious relationship between these skew Kostka-Foulkes polynomials and the polynomials

$$
\sum_{T \in \operatorname{SSYT}\left(\nu^{\prime}, \alpha\right)} q^{\text {charge }_{\mu^{\prime}}(T)}
$$

appearing in (25).

5.2. CSP ON SKEW SPECIALIZED MACDONALd POLYNOMials. We can generalize Theorem 3.2 to the skew setting. We let $\phi$ act on $\operatorname{COF}(n \lambda / n \mu, m)$ as before, by cyclically shifting each block of $n$ consecutive columns one step to the right, followed by rearranging the entries in each column such that a (unique) specialized Macdonald filling is obtained. Again, $\langle\phi\rangle$ is a cyclic group of order $n$.

THEOREM 5.10. For every skew shape $\lambda / \mu$ and $n \geqslant 1$, the triple

$$
\left(\operatorname{COF}(n \lambda / n \mu, m),\langle\phi\rangle, \mathrm{E}_{n \lambda / n \mu}\left(1^{m} ; q, 0\right)\right)
$$

exhibits the cyclic sieving phenomenon. Furthermore, the family

$$
\left\{\left(\operatorname{COF}(n \lambda / n \mu, m),\langle\phi\rangle, \mathrm{E}_{n \lambda / n \mu}\left(1^{m} ; q, 0\right)\right)\right\}_{n=1}^{\infty}
$$

is Lyndon-like, as described in Definition 2.9. 
Proof. The number of descents between two adjacent columns of the same height only depends on the set of entries in the two columns, and this is proved in [48, Thm. 3.15]. A priori, one would not expect this as the actual positions of the entries depend on all previous columns in a coinversion-free filling.

Now consider the blocks of columns, where each block consists of $n$ consecutive columns (of the same height). Descents involving two entries from different blocks only contribute with a multiple of $n$ to the major index. Hence, in order to determine the major index mod $n$ of a filling, it suffices to examine the columns in each block separately. Let $\nu$ be the partition such that the parts of $\nu^{\prime}$ are given by the multiset $\left\{\lambda_{i}^{\prime}-\mu_{i}^{\prime}: i=1,2, \ldots\right\}$. By the previous observations,

$$
\mathrm{E}_{n \lambda / n \mu}\left(1^{m} ; q, 0\right) \equiv \mathrm{E}_{n \nu}\left(1^{m} ; q, 0\right) \quad \bmod \left(q^{n}-1\right) .
$$

It is then straightforward to use the same arguments as in the non-skew case, Theorem 3.2 , to finish the proof.

Note that Theorem 4.3 can be generalized to the skew setting using a similar argument — we leave the details to the reader.

\section{Crystal operators on wOrds And SSYT}

We now recall some minimal background on crystal operators (in the sense of Kashiwara and Stembridge) on words and semistandard Young tableaux, see [14, 42] for more background.

The operators $\tilde{e}_{i}, \tilde{f}_{i}: \mathbb{N}^{k} \rightarrow \mathbb{N}^{k} \cup\{\varnothing\}$ are defined as follows. Given a word $w \in \mathbb{N}^{k}$ consider the subword $w_{i}$ consisting only of the letters $i$ and $i+1$. Replace each instance of $i$ with a right bracket and each $i+1$ with a left bracket. Remove all pairs of matching brackets and consider the remaining unmatched brackets, which now consist of $a$ rightbrackets and $b$ left-brackets. These brackets correspond to a subword $w^{\prime}$ of the form $i^{a}(i+1)^{b}$ in $w$. The operator $\tilde{e}_{i}$ acting on $w$ turns the leftmost $i+1$ of $w^{\prime}$ into an $i$, if such an entry exists, otherwise, $\tilde{e}_{i}(w):=\varnothing$. Similarly, $\tilde{f}_{i}$ acting on $w$ turns the rightmost $i$ in $w^{\prime}$ into an $i+1$, if such an entry exists, otherwise, $\tilde{f}_{i}(w):=\varnothing$. For example,

$$
\tilde{e}_{1}(2,1,3,1,2,4,2,1,1,3,1, \underline{2}, 3,2,1,2,1)=2,1,3,1,2,4,2,1,1,3,1,1,3,2,1,2,1 .
$$

The operator $\tilde{e}_{i}$ is a crystal raising operator while $\tilde{f}_{i}$ is a crystal lowering operator. The operators also act on semistandard Young tableaux by acting on the reading word. We define a graph structure on words (or semistandard Young tableaux) by having a labeled directed edge $u \rightarrow v$ with label $i$ if $\tilde{f}_{i}(u)=v$. Examples on such components are given in Figure 9.

6.1. Crystal operators on COF And RSK. We shall now define crystal operators on the set $\operatorname{COF}(\lambda / \mu)$. These operators are considered in [48], and the non-skew case was recently considered by S. Assaf and N. Gonzáles [9], where the authors prove that they are indeed crystal operators. Here, we take a slightly different route and define the operators on biwords instead - it is straightforward to verify that our definitions are equivalent with theirs. These crystal biwords are closely related to the biwords we have seen previously.

Definition 6.1 (Crystal operators on fillings). Let $F \in \operatorname{COF}(\lambda / \mu)$ and define the crystal biword $\tilde{W}$, with entry $\left(\begin{array}{l}j \\ c\end{array}\right)$ appearing in $\tilde{W}$ if and only if there is a box with value $j$ in column $c$. The entries in $\tilde{W}$ are then sorted decreasingly, primarily on the bottom row entry, see Figure 10. Note that the map to $\tilde{W}$ is invertible if $\lambda / \mu$ is fixed. We then define $\tilde{e}_{i}(F)$, and $\tilde{f}_{i}(F)$ as the result when applying $\tilde{e}_{i}$ and $\tilde{f}_{i}$, respectively, on the first row of $\tilde{W}$. 

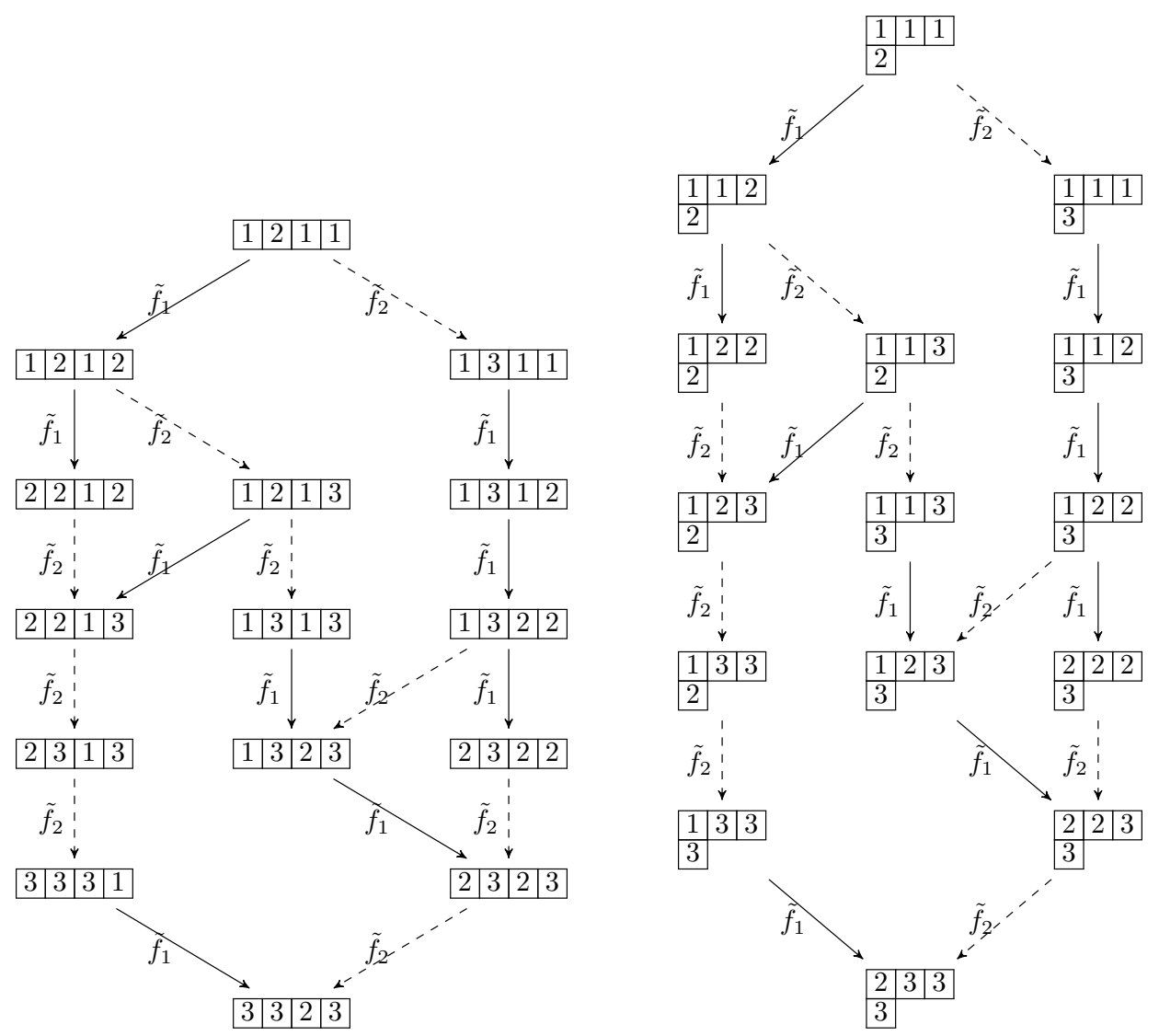

Figure 9. A crystal graph on words and one on SSYT.

For the subset of coinversion-free fillings $F$ with maj $(F)=0$, these operators are essentially a generalization of the raising and lowering operators defined on semistandard Young tableaux.

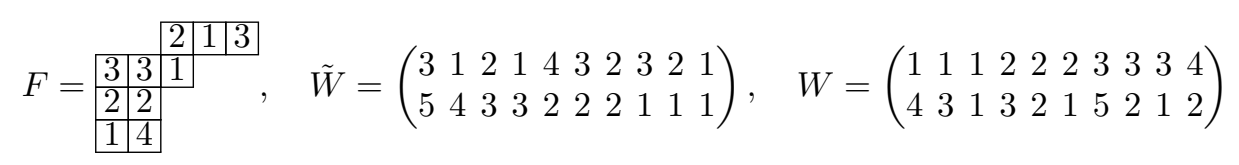

Figure 10. A skew specialized Macdonald filling $F$, the corresponding crystal biword $\tilde{W}$ and the biword $W$ used for RSK. Note that the only difference between $\tilde{W}$ and $W$ is the ordering of the entries.

The following theorem was proven independently by S. Assaf and N. Gonzáles [9] and the second author [48].

TheOrem 6.2 (See [48, Thm. 3.19], [9]). The operators $\tilde{e}_{i}$ and $\tilde{f}_{i}$ preserve major index. That is, suppose that $F$ and $F^{\prime}$ are in $\operatorname{COF}(\lambda / \mu)$ and that $\tilde{e}_{i}(F), \tilde{f}_{i}\left(F^{\prime}\right) \neq \varnothing$. Then

$$
\operatorname{maj}\left(\tilde{e}_{i}(F)\right)=\operatorname{maj}(F) \text { and } \operatorname{maj}\left(\tilde{f}_{i}\left(F^{\prime}\right)\right)=\operatorname{maj}\left(F^{\prime}\right) .
$$

Furthermore, $F, \tilde{e}_{i}(F)$ and $\tilde{f}_{i}(F)$ differ only at boxes with entries $i$ and $i+1$. 
In Theorem 6.4 below we prove that the operators $\tilde{e}_{i}$ and $\tilde{f}_{i}$ define proper crystal graphs on the set $\operatorname{COF}(\lambda / \mu)$. See Figure 11 for examples of crystal graphs on coinversion-free fillings.
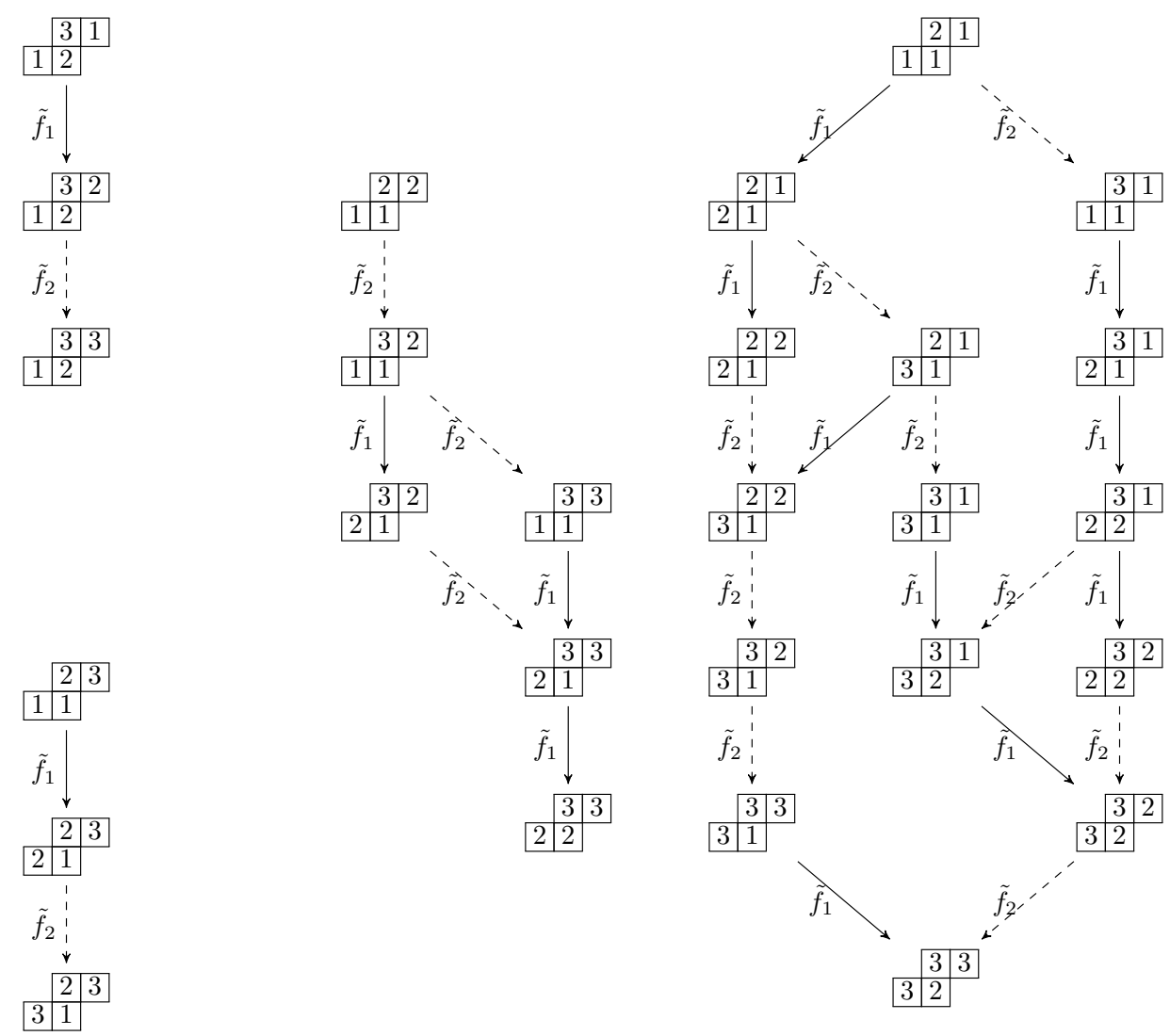

FiguRE 11. The crystal structure on skew coinversion-free fillings of shape $\lambda / \mu$ with $\lambda=(3,2)$ and $\mu=(1)$ in 3 variables. Notice that the large component is isomorphic to the crystal graphs in Figure 9.

There is an important interaction between the crystal operators and the RSK correspondence, as we shall see in the following example.

ExAmple 6.3. Suppose $F \in \operatorname{COF}(\lambda)$ with biword $W$

$$
W=\left(\begin{array}{llllllllll}
1 & 1 & 1 & 2 & 2 & 2 & 3 & 3 & 3 & 4 \\
4 & 3 & 1 & 3 & 2 & 1 & 5 & 2 & 1 & 2
\end{array}\right)
$$

The crystal biword is

$$
\tilde{W}=\left(\begin{array}{llllllllll}
3 & 1 & 2 & 1 & 4 & 3 & 2 & 3 & 2 & 1 \\
5 & 4 & 3 & 3 & 2 & 2 & 2 & 1 & 1 & 1
\end{array}\right)
$$

Apply $\tilde{e}_{1}$ on the top row. We have the subword 121221 which turns into 121121 under $\tilde{e}_{1}$. The resulting biword after applying $\tilde{e}_{1}$ on the top row is therefore

$$
\left(\begin{array}{llllllllll}
3 & 1 & 2 & 1 & 4 & 3 & 1 & 3 & 2 & 1 \\
5 & 4 & 3 & 3 & 2 & 2 & 2 & 1 & 1 & 1
\end{array}\right)
$$


Finally, the columns are sorted, and we obtain the biword $W^{\prime}$ that corresponds to $\tilde{e}_{1}(F)$ :

$$
\tilde{e}_{1}(F) \leftrightarrow\left(\begin{array}{llllllllll}
1 & 1 & 1 & 1 & 2 & 2 & 3 & 3 & 3 & 4 \\
4 & 3 & 2 & 1 & 3 & 1 & 5 & 2 & 1 & 2
\end{array}\right) .
$$

If we now perform RSK on $W$ and $W^{\prime}$ we see in Figure 12 that $\tilde{e}_{1}$ has a predictable effect on the corresponding insertion and recording tableau, see Theorem 6.4.

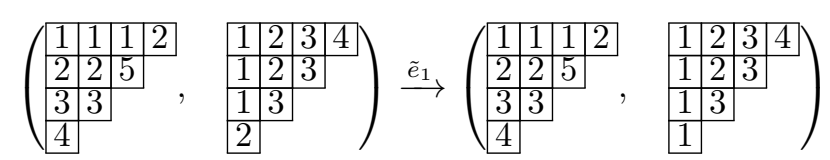

FiguRE 12. An example of the raising operator at the level of RSK. The pairs of tableaux correspond to the two biwords in Example 6.3.

TheOREM 6.4. Let $F \in \operatorname{COF}(\lambda / \mu)$ and suppose $\tilde{e}_{i}(F) \neq \varnothing$. Then $\operatorname{ins}(F)=\operatorname{ins}\left(\tilde{e}_{i}(F)\right)$ and $\tilde{e}_{i}(\operatorname{rec}(F))=\operatorname{rec}\left(\tilde{e}_{i}(F)\right)$. Stated equivalently on biwords: Let $W$ be a biword and let $\tilde{W}$ be its entries reordered such that it is a crystal biword. Suppose $\tilde{e}_{i}(\tilde{W}) \neq \varnothing$, and that $W^{\prime}$ is the biword corresponding to $\tilde{e}_{i}(\tilde{W})$. Then $\operatorname{ins}(W)=\operatorname{ins}\left(W^{\prime}\right)$ and $\tilde{e}_{i}(\operatorname{rec}(W))=\operatorname{rec}\left(W^{\prime}\right)$. The analogous statements for $\tilde{f}_{i}$ also hold.

Proof sketch. The fact that $\operatorname{ins}(F)=\operatorname{ins}\left(\tilde{e}_{i}(F)\right)$ follows from properties of the classical RSK algorithm.

The second property requires some more work, but every step is a routine transformation using known properties of various versions of RSK. First restate the property to the analogous statement about the dual RSK insertion algorithm (using column insertion), see [27, Sec. 4.3]. One can then use that dual RSK and classical RSK are related in a simple manner (see e.g. [15, Prop. 2.3.14]) and reduce the problem further to the case of the classical RSK. For classical RSK, the interaction with crystal operators is well-documented, see e.g. [14, 28, 42].

Using the bijection in Theorem 6.4, we see that the set $\operatorname{COF}(\lambda / \mu)$ is indeed a crystal graph under the raising and lowering operators, since we have an equivariant bijection with crystals and crystal operators on semistandard tableaux. We remark that S. Assaf and N. Gonzáles [9] proved the same result in the non-skew case by verifying the local characterization axioms introduced by Stembridge, see [46].

We shall now briefly discuss an application of the crystal operators. Using the crystal operators $\tilde{e}_{i}$ and $\tilde{f}_{i}$, we can define involutions on $\operatorname{COF}(\lambda / \mu)$. For a coinversionfree filling $F$, denote $m_{i}=m_{i}(F)$ the number of $i$-entries of $F$.

Definition 6.5. For $i \in \mathbb{N}$, define the operators $\tilde{s}_{i}$ on $\operatorname{COF}(\lambda / \mu)$ by letting

$$
\tilde{s}_{i}(F):= \begin{cases}\left(\tilde{e}_{i}\right)^{m_{i+1}-m_{i}}(F) & \text { if } m<m_{i+1} \\ \left(\tilde{f}_{i}\right)^{m-m_{i+1}}(F) & \text { if } m_{i}>m_{i+1} \\ F & \text { if } m_{i}=m_{i+1} .\end{cases}
$$

Restricted to the set of coinversion-free fillings with maj $=0$, the operators $\tilde{s}_{i}$ are essentially the famous Lascoux-Schützenberger involutions [30]. The difference is that the elements with maj $=0$ have weakly decreasing rows and strictly increasing columns as opposed to the weakly increasing in rows and strictly increasing columns for in semistandard Young tableaux.

It is clear by Theorem 6.2 that the operators $\tilde{s}_{i}$ are maj-preserving involutions. Furthermore, if $F \in \operatorname{COF}(\lambda / \mu)$ and $\operatorname{wt}(F)=\left(w_{1}, \ldots, w_{\ell}\right)$, then $\operatorname{wt}\left(\tilde{s}_{i}(F)\right)=$ 
$\left(w_{1}, \ldots, w_{i+1}, w_{i}, \ldots, w_{\ell}\right)$. This yields yet another proof that $\mathrm{E}_{\lambda / \mu}(\mathbf{x} ; q, 0)$ is symmetric. In fact, it follows from general theory of crystals that the operators $\tilde{s}_{1}, \tilde{s}_{2}$,

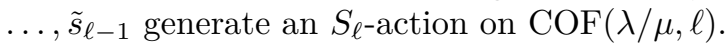

\section{SCHUR EXPANSION OF CERTAIN VERTICAL-STRIP LLT POLYNOMIALS}

In this section, we briefly sketch that $\mathrm{E}_{\lambda / \mu}(\mathbf{x} ; q, 0)$ sometimes is a vertical strip LLT polynomial, up to a power of $q$. As a consequence, we therefore obtain an explicit formula for the Schur expansion of these particular LLT polynomials. Hence, we provide a new family of LLT polynomials with a combinatorial Schur expansion, not covered by previous results. We note that it is a major open problem in general to describe the LLT polynomials in the Schur basis.

Definition 7.1 (As in [21]). Let $\boldsymbol{\nu}$ be a k-tuple of skew Young diagrams. Given such a tuple, we let $\operatorname{SSYT}(\boldsymbol{\nu})=\operatorname{SSYT}\left(\boldsymbol{\nu}^{1}\right) \times \operatorname{SSYT}\left(\boldsymbol{\nu}^{2}\right) \times \cdots \times \operatorname{SSYT}\left(\boldsymbol{\nu}^{k}\right)$ where $\operatorname{SSYT}(\lambda / \mu)$ is the set of skew semistandard Young tableaux of shape $\lambda / \mu$. Given $T=\left(T^{1}, T^{2}, \ldots, T^{k}\right) \in \operatorname{SSYT}(\boldsymbol{\nu})$, let $\mathbf{x}^{T}:=\mathbf{x}^{T^{1}} \cdots \mathbf{x}^{T^{k}}$ where $\mathbf{x}^{T^{i}}$ is the same monomial weight of $T^{i}$ as for Schur polynomials. Given a cell $u=(r, c)$ (row, column) in a skew diagram, the content is defined as $c(u):=c-r$.Entries $T^{i}(u)>T^{j}(v)$ in a tuple form an inversion if and only if

$$
i<j \text { and } c(u)=c(v), \quad \text { or } \quad i>j \text { and } c(u)=c(v)-1 .
$$

The LLT polynomial associated with the $k$-tuple $\boldsymbol{\nu}$ is given by

$$
\operatorname{LLT}_{\boldsymbol{\nu}}(\mathbf{x} ; q)=\sum_{T \in \operatorname{SSYT}(\boldsymbol{\nu})} q^{\operatorname{inv}(T)} \mathbf{x}^{T}
$$

where $\operatorname{inv}(T)$ is the total number of inversions appearing in $T$. One can show that $\operatorname{LLT}_{\boldsymbol{\nu}}(\mathbf{x} ; q)$ is a symmetric function, see [21] or [4] for short proofs.

LLT polynomials such that each $\nu^{j}$ is a skew shape of the form $1^{a} / 1^{b}$ with $a \geqslant b$ are called vertical strip LLT polynomials. Given a $k$-tuple $\boldsymbol{\nu}$, we let $\operatorname{mininv}(\boldsymbol{\nu})$ be the minimum number of inversions obtainable over all fillings. That is,

$$
\operatorname{mininv}(\boldsymbol{\nu}):=\min _{T \in \operatorname{SSYT}(\boldsymbol{\nu})} \operatorname{inv}(T) .
$$

EXAMPLE 7.2. A $k$-tuple of skew shapes is traditionally illustrated using the French convention where box $(1,1)$ of each shape $\boldsymbol{\nu}^{i}$ is placed on the line $y=x$ with content 0 , and Cartesian coordinates are used. Below, we illustrate an element

$$
T \in \operatorname{SSYT}\left(1^{3} / \varnothing\right) \times \operatorname{SSYT}\left(1^{3} / 1^{1}\right) \times \operatorname{SSYT}\left(1^{2} / 1^{1}\right) \times \operatorname{SSYT}\left(1^{3} / \varnothing\right)
$$

which appears when computing the vertical-strip $\operatorname{LLT}$ polynomial $\operatorname{LLT}_{\boldsymbol{\nu}}(\mathbf{x} ; q)$ for $\boldsymbol{\nu}=$ $(111 / \varnothing, 111 / 1,11 / 1,111 / \varnothing)$.

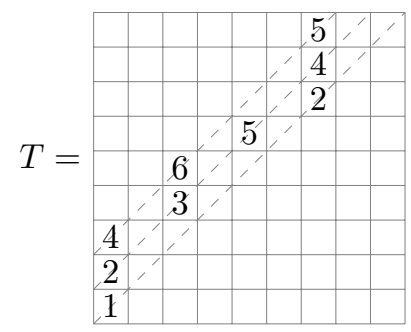

There are two inversions involving boxes $u$ and $v$ where $c(u)=c(v)$ and six inversions for which $c(u)=c(v)-1$. Hence, $T$ contributes with $q^{8} x_{1} x_{2}^{2} x_{3} x_{4}^{2} x_{5}^{2} x_{6}$. The full LLT 
polynomial $\operatorname{LLT}_{\boldsymbol{\nu}}(\mathbf{x} ; q)$ in the Schur basis is given by

$$
\begin{aligned}
& q^{8} \mathrm{~s}_{333}+q^{7} \mathrm{~s}_{432}+\left(q^{9}+q^{10}+q^{11}\right) \mathrm{s}_{3222}+\left(q^{8}+2 q^{9}+q^{10}\right) \mathrm{s}_{3321}+\left(q^{8}+q^{9}\right) \mathrm{s}_{4221} \\
& +q^{8} \mathrm{~s}_{4311}+\left(q^{10}+q^{11}+q^{12}+q^{13}\right) \mathrm{s}_{22221}+\left(q^{9}+3 q^{10}+2 q^{11}+q^{12}\right) \mathrm{s}_{32211} \\
& +\left(q^{9}+q^{10}+q^{11}\right) \mathrm{s}_{33111}+\left(q^{9}+q^{10}\right) \mathrm{s}_{42111}+\left(2 q^{11}+2 q^{12}+q^{13}+q^{14}\right) \mathrm{s}_{222111} \\
& +\left(q^{10}+2 q^{11}+2 q^{12}+q^{13}\right) \mathrm{s}_{321111}+q^{11} \mathrm{~s}_{411111}+\left(q^{12}+2 q^{13}+q^{14}+q^{15}\right) \mathrm{s}_{2211111} \\
& +\left(q^{12}+q^{13}+q^{14}\right) \mathrm{s}_{3111111}+\left(q^{14}+q^{15}+q^{16}\right) \mathrm{s}_{21111111}+q^{17} \mathrm{~s}_{111111111} .
\end{aligned}
$$

As $q^{7}$ is the lowest power of $q$ that appear in the expansion, we must have that $\operatorname{mininv}(\boldsymbol{\nu})=7$.

The current state-of-the-art regarding combinatorial proofs of Schur positivity of LLT polynomials is as follows.

- When all shapes in $\boldsymbol{\nu}$ are non-skew, the coefficients in the Schur basis are known to be certain parabolic Kazhdan-Lusztig polynomials, see [31]. Hence, the coefficients are in $\mathbb{N}[q]$. In particular, this case contains the Hall-Littlewood symmetric functions.

- Whenever the $k$-tuple of shapes $\boldsymbol{\nu}$ consists of at most 3 shapes, all avoiding an arrangement of $2 \times 2$-boxes (that is, they are ribbons), Schur positivity is given by a combinatorial formula, see J. Blasiak [13].

- A few other cases when each shape in $\boldsymbol{\nu}$ is a single box is given in [23].

THEOREM 7.3. Let $\lambda / \mu$ be a skew shape such that no column contains more than two boxes. Then

$$
\mathrm{E}_{\lambda^{\prime} / \mu^{\prime}}(\mathbf{x} ; q, 0)=q^{-\operatorname{mininv}(\boldsymbol{\nu})} \operatorname{LLT}_{\boldsymbol{\nu}}(\mathbf{x} ; q)
$$

where $\boldsymbol{\nu}_{j}$ is the vertical strip $\left(\lambda_{j}\right) /\left(\mu_{j}\right)$.

Proof sketch. For the modified Macdonald polynomials $\tilde{\mathrm{H}}_{\lambda}(\mathbf{x} ; q, t)$, we have the symmetry $\tilde{\mathrm{H}}_{\lambda}(\mathbf{x} ; q, t)=\tilde{\mathrm{H}}_{\lambda^{\prime}}(\mathbf{x} ; t, q)$. This interchanges the rôle of inversion triples and major index, see [20]. This relationship extends to modified Macdonald polynomials indexed by skew shapes $\lambda / \mu$ as long as each column contains at most two boxes, see J. Bandlow, [10, Thm. 5].

There is a correspondence between inversion triples and inversions that appearing definition of LLT polynomials. In [21, Eq. (23)], the authors provide (via a straightforward bijective argument) an expansion of the form

$$
\tilde{\mathrm{H}}_{\lambda}(\mathbf{x} ; q, t)=\sum_{D} q^{\operatorname{maj}(D)} t^{-\operatorname{stat}(D)} \operatorname{LLT}_{\nu(D)}(\mathbf{x} ; t),
$$

where the sum runs over all subsets (possible descents) of boxes $(i, j)$ with $i>1$ of the diagram $\lambda$. In particular, the coefficient of the terms maximizing the major index a vertical-strip LLT polynomial. This expansion has a natural extension to skew shapes and one can check that $\operatorname{stat}(\cdot)$ corresponds to $\operatorname{mininv}(\cdot)$ for the highest-degree term. Combining all these observations we have

$$
\mathrm{E}_{\lambda^{\prime} / \mu^{\prime}}(\mathbf{x} ; q, 0)=\left[t^{*}\right] \tilde{\mathrm{H}}_{\lambda / \mu}(\mathbf{x} ; q, t)=\left[t^{*}\right] \tilde{\mathrm{H}}_{\lambda^{\prime} / \mu^{\prime}}(\mathbf{x} ; t, q)=q^{-\operatorname{mininv}(\boldsymbol{\nu})} \operatorname{LLT}_{\boldsymbol{\nu}}(\mathbf{x} ; q) .
$$

The first identity is due to (22). The second identity is the tricky part and relies on [10]. The third identity is a simple consequence of (29).

EXAMPLE 7.4. We illustrate Theorem 7.3 in the case $\lambda / \mu=4431 / 31$. The skew shape $\lambda / \mu$ is illustrated in (30) where we have labeled the boxes row by row, from right to left in each row. The corresponding $k$-tuple of vertical strips is shown to the right. The labeling has the property that it maps inversion pairs in the filling to the right, to inversions in the LLT diagram, see [21] for details. 

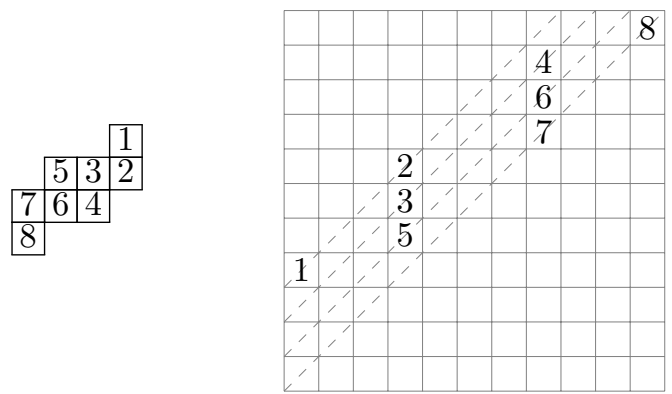

Notice that no column contains more than two boxes so the conditions in the theorem applies. The $k$-tuple $\boldsymbol{\nu}$ is $1111 / 111,1111 / 1,111 / \varnothing, 1 / \varnothing$, and it is easy (for a computer) to check that

$$
\begin{aligned}
\mathrm{E}_{\lambda^{\prime} / \mu^{\prime}}(\mathbf{x} ; q, 0)= & \mathrm{s}_{332}+\mathrm{s}_{422}+\left(1+q^{2}\right) \mathrm{s}_{2222}+(2+2 q) \mathrm{s}_{3221}+\mathrm{s}_{3311}+\mathrm{s}_{4211} \\
& +\left(3 q+q^{2}\right) \mathrm{s}_{22211}+4 q \mathrm{~s}_{32111}+q \mathrm{~s}_{41111} \\
& 4 q^{2} \mathrm{~s}_{221111}+3 q^{2} \mathrm{~s}_{311111} \\
& +3 q^{3} \mathrm{~s}_{2111111}+q^{4} \mathrm{~s}_{1111111}
\end{aligned}
$$

and that this is also equal to $q^{-1} \operatorname{LLT}_{\boldsymbol{\nu}}(\mathbf{x} ; q)$.

As a final check, we verify one of the coefficients with the combinatorial formula. Using the notation in Theorem 5.7, $\alpha=1331$. The term $(2+2 q) \mathrm{s}_{3221}$ then arises from the four semistandard tableaux

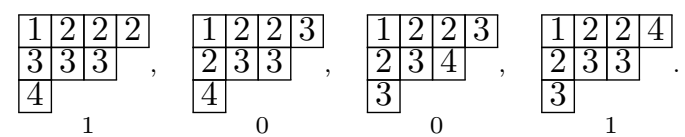

where the value of $\operatorname{charge}_{31}(w)=\operatorname{charge}(w \cdot 2111)$ is shown under each tableau.

Acknowledgements. The authors would like to thank Svante Linusson and Samu Potka for helpful discussions. We also thank Jim Haglund for suggesting to look at the connection with LLT polynomials and the relevance of [10]. We are very grateful for the suggestions and excellent job done by the referees. The first author was funded by the Swedish Research Council (Vetenskapsrådet), grant 2015-05308.

\section{REFERENCES}

[1] Connor Ahlbach and Joshua P. Swanson, Refined cyclic sieving on words for the major index statistic, European J. Combin. 73 (2018), 37-60.

[2] Per Alexandersson, Non-symmetric Macdonald polynomials and Demazure-Lusztig operators, Sémin. Lothar. Comb. 76 (2019), B76d (27 pages).

[3] Per Alexandersson, Svante Linusson, and Samu Potka, The cyclic sieving phenomenon on circular Dyck paths, Electron. J. Combin. 26 (2019), no. 4, Paper 4.16 (32 pages).

[4] Per Alexandersson and Greta Panova, LLT polynomials, chromatic quasisymmetric functions and graphs with cycles, Discrete Math. 341 (2018), no. 12, 3453-3482.

[5] Per Alexandersson, Stephan Pfannerer, Martin Rubey, and Joakim Uhlin, Skew characters and cyclic sieving, https://arxiv.org/abs/2004.01140, 2020.

[6] Per Alexandersson and Mehtaab Sawhney, A major-index preserving map on fillings, Electron. J. Combin. 24 (2017), no. 4, Paper 4.3 (30 pages).

[7] - Properties of non-symmetric Macdonald polynomials at $q=1$ and $q=0$, Ann. Comb. 23 (2019), no. 2, 219-239.

[8] Sami Assaf, Nonsymmetric Macdonald polynomials and a refinement of Kostka-Foulkes polynomials, Trans. Amer. Math. Soc. 370 (2018), no. 12, 8777-8796.

[9] Sami Assaf and Nicolle S. González, Crystal graphs, key tabloids, and nonsymmetric Macdonald polynomials, Sém. Lothar. Combin. 80B (2018), Art. 81 (12 pages), 30th International Conference on Formal Power Series and Algebraic Combinatorics. 
[10] Jason Bandlow, Combinatorics of Macdonald polynomials and extensions, Ph.D. thesis, UC San Diego, 2007, https://escholarship.org/uc/item/5zd262sp.

[11] Max Bennett, Blake Madill, and Anna Stokke, Jeu-de-taquin promotion and a cyclic sieving phenomenon for semistandard hook tableaux, Discrete Math. 319 (2014), 62-67.

[12] Andrew Berget, Sen-Peng Eu, and Victor Reiner, Constructions for cyclic sieving phenomena, SIAM J. Discrete Math. 25 (2011), no. 3, 1297-1314.

[13] Jonah Blasiak, Haglund's conjecture on 3-column Macdonald polynomials, Math. Z. 283 (2016), no. 1-2, 601-628.

[14] Daniel Bump and Anne Schilling, Crystal bases. Representations and combinatorics, Hackensack, NJ: World Scientific, 2017 (English).

[15] Lynne M. Butler, Subgroup lattices and symmetric functions, vol. 112, Mem. Amer. Math. Soc., no. 539, American Mathematical Society, 1994.

[16] Ivan Cherednik, Nonsymmetric Macdonald polynomials, Internat. Math. Res. Notices (1995), no. 10, 483-515.

[17] Jacques Désarménien, Bernard Leclerc, and Jean-Yves Thibon, Hall-Littlewood functions and Kostka-Foulkes polynomials in representation theory, Sém. Lothar. Combin. 32 (1994), Art. B32c (38 pages).

[18] William Fulton, Young tableaux: With applications to representation theory and geometry, London Mathematical Society Student Texts, vol. 35, Cambridge University Press, 1997.

[19] Ofir Gorodetsky, q-congruences, with applications to supercongruences and the cyclic sieving phenomenon, Int. J. Number Theory 15 (2019), no. 9, 1919-1968.

[20] James Haglund, The q,t-Catalan numbers and the space of diagonal harmonics, University lecture series, vol. 41, American Mathematical Society, 2007.

[21] James Haglund, Mark Haiman, and Nicholas A. Loehr, A combinatorial formula for Macdonald polynomials, J. Amer. Math. Soc. 18 (2005), no. 3, 735-761.

[22] _ A combinatorial formula for nonsymmetric Macdonald polynomials, Amer. J. Math. 130 (2008), no. 2, 359-383.

[23] JiSun Huh, Sun-Young Nam, and Meesue Yoo, Melting lollipop chromatic quasisymmetric functions and Schur expansion of unicellular LLTpolynomials, Discrete Math. 343 (2020), no. 3, 111728 (21 pages).

[24] Ryan Kaliszewski and Jennifer Morse, Colorful combinatorics and Macdonald polynomials, European J. Combin. 81 (2019), 354-377, https://doi.org/10.1016/j.ejc.2019.05.006.

[25] Anatol N. Kirillov, Anne Schilling, and Mark Shimozono, Various representations of the generalized kostka polynomials, in The Andrews Festschrift, Springer Berlin Heidelberg, Berlin, Heidelberg, 2001, pp. 209-226.

[26] Friedrich Knop and Siddhartha Sahi, A recursion and a combinatorial formula for Jack polynomials, Invent. Math. 128 (1997), no. 1, 9-22.

[27] Christian Krattenthaler, Growth diagrams, and increasing and decreasing chains in fillings of Ferrers shapes, Adv. in Appl. Math. 37 (2006), no. 3, 404-431, https://doi.org/10.1016/j. aam.2005.12.006.

[28] Alain Lascoux, Double crystal graphs, in Studies in memory of Issai Schur (Chevaleret/Rehovot, 2000), Progr. Math., vol. 210, Birkhäuser Boston, Boston, MA, 2003, pp. 95-114.

[29] Alain Lascoux, Bernard Leclerc, and Jean-Yves Thibon, Green polynomials and Hall-Littlewood functions at roots of unity, European J. Combin. 15 (1994), no. 2, 173-180.

[30] Alain Lascoux and Marcel-Paul Schützenberger, Sur une conjecture de H. O. Foulkes, C. R. Acad. Sci. Paris Sér. A-B 286 (1978), no. 7, A323-A324.

[31] Bernard Leclerc and Jean-Yves Thibon, Littlewood-Richardson coefficients and KazhdanLusztig polynomials, Combinatorial methods in representation theory (Kyoto, 1998), Adv. Stud. Pure Math., vol. 28, Kinokuniya, Tokyo, 2000, pp. 155-220.

[32] Ian G. Macdonald, Symmetric functions and Hall polynomials, second ed., Oxford Mathematical Monographs, The Clarendon Press, Oxford University Press, New York, 1995, With contributions by A. Zelevinsky, Oxford Science Publications.

[33] Young-Tak Oh and Euiyong Park, Crystals, semistandard tableaux and cyclic sieving phenomenon, Electron. J. Combin. 26 (2019), no. 4, Paper 4.39 (19 pages).

[34] Eric M. Opdam, Harmonic analysis for certain representations of graded Hecke algebras, Acta Math. 175 (1995), no. 1, 75-121.

[35] Victor Reiner, Dennis Stanton, and Dennis E. White, The cyclic sieving phenomenon, J. Combin. Theory Ser. A 108 (2004), no. 1, 17-50.

[36] Brendon Rhoades, Cyclic sieving, promotion, and representation theory, J. Combin. Theory Ser. A 117 (2010), no. 1, 38-76. 
[37] _ Hall-Littlewood polynomials and fixed point enumeration, Discrete Math. 310 (2010), no. $4,869-876$

[38] David B. Rush, Cyclic sieving and plethysm coefficients, Trans. Amer. Math. Soc. 371 (2019), no. 2, 923-947.

[39] Bruce E. Sagan, Congruence properties of q-analogs, Adv. Math. 95 (1992), no. 1, 127-143.

[40] - The cyclic sieving phenomenon: a survey, in Surveys in combinatorics 2011, London Math. Soc. Lecture Note Ser., vol. 392, Cambridge Univ. Press, Cambridge, 2011, pp. 183-233.

[41] Linhui Shen and Daping Weng, Cyclic sieving and cluster duality for Grassmannian, https: //arxiv.org/abs/1803.06901, 2018

[42] Mark Shimozono, Crystals for dummies, Online, 2005, https://www.aimath.org/WwN/kostka/ crysdumb.pdf.

[43] Mark Shimozono and Jerzy Weyman, Graded characters of modules supported in the closure of a nilpotent conjugacy class, European J. Combin. 21 (2000), no. 2, 257-288.

[44] Neil J. A. Sloane, The On-Line Encyclopedia of Integer Sequences, Online, 2019, https://oeis. org.

[45] Richard P. Stanley, Enumerative Combinatorics: Volume 2, first ed., Cambridge Studies in Advanced Mathematics, vol. 62, Cambridge University Press, 2001.

[46] John R. Stembridge, A local characterization of simply-laced crystals, Trans. Amer. Math. Soc. 355 (2003), no. 12, 4807-4823.

[47] Geanina Tudose and Mike Zabrocki, A q-analog of Schur's Q-functions, in Algebraic Combinatorics and Quantum Groups (Naihuan Jing, ed.), World Scientific, 2003, pp. 135-161.

[48] Joakim Uhlin, Combinatorics of Macdonald polynomials and cyclic sieving, M.s thesis, KTH, Mathematics (Div.), January 2019, http://kth.diva-portal.org/smash/record.jsf? pid=diva $2 \% 3$ A 1282825 .

Per Alexandersson, Dept. of Mathematics, Royal Institute of Technology, SE-100 44 Stockholm, Sweden

E-mail : per.w.alexandersson@gmail.com

JoAkim Uhlin, Dept. of Mathematics, Royal Institute of Technology, SE-100 44 Stockholm, Sweden E-mail : joakim_uhlin@hotmail.com 